\title{
An Implementation of an Aeroacoustic Prediction Model for Broadband Noise from a Vertical Axis Wind Turbine Using a CFD Informed Methodology
}

\author{
J.D.M. Botha ${ }^{\mathrm{a}, 1}$, A Shahroki ${ }^{\mathrm{b}}$,H. Rice ${ }^{\mathrm{a}}$ \\ ${ }^{a}$ Trinity College Dublin, The University of Dublin, College Green, Dublin 2, Ireland \\ ${ }^{b}$ Department of Mechanical Engineering, University of Sheffield, Sheffield, UK
}

\begin{abstract}
This paper presents an enhanced method for predicting aerodynamically generated broadband noise produced by a Vertical Axis Wind Turbine (VAWT). The method improves on existing work for VAWT noise prediction and incorporates recently developed airfoil noise prediction models. Inflow-turbulence and airfoil self-noise mechanisms are both considered. Airfoil noise predictions are dependent on aerodynamic input data and time dependent Computational Fluid Dynamics (CFD) calculations are carried out to solve for the aerodynamic solution. Analytical flow methods are also benchmarked against the CFD informed noise prediction results to quantify errors in the former approach. Comparisons to experimental noise measurements for an existing turbine are encouraging. A parameter study is performed and shows the sensitivity of overall noise levels to changes in inflow velocity and inflow turbulence. Noise sources are characterised and the location and mechanism of the primary sources is determined, inflow-turbulence noise is seen to be the dominant source. The use of CFD calculations is seen to improve the accuracy of noise predictions when compared to the analytic flow solution as well as showing that, for inflow-turbulence noise sources, blade generated turbulence dominates the atmospheric inflow turbulence.
\end{abstract}

Keywords: Vertical Axis Wind Turbine, Computational Aeroacoustics, Computational Fluid Dynamics, Broadband Noise, Airfoil Noise

\section{Introduction}

Small Vertical Axis Wind Turbines (VAWT) can be easily integrated into urban and peri-urban environments as a means to generate power in these locations. There has been very little work reported to quantify their noise output as it is generally considered that small VAWT's should be quiet enough given that their

5 deployment in urban areas means that their noise output rarely exceeds that of their surroundings [1. However, early stage noise source prediction is still necessary during turbine design in order to approve designs for integration into urban environments.

Small Wind Turbines (SWT's) are defined, according to IEC 61400-2:2013, an International Standard published by the International Electrotechnical Commission regarding wind turbines, as having a swept area 10 of less than $200 \mathrm{~m}^{2}$. This corresponds to a Horizontal Axis Wind Turbine (HAWT) with a diameter of around $16 \mathrm{~m}$. For a VAWT with an equivalent swept area, this corresponds to any machine with a diameter of less than $5 \mathrm{~m}$ and span of $10 \mathrm{~m}$.

Preliminary design of SWT's almost exclusively incorporates the use of low- or reduced-order prediction tools to make early performance estimates. Popular design tools such as QBlade 2] utilise methods such as the 15 Double-Multiple Streamtube (DMS) 3] approach for aerodynamic performance estimates. Noise estimates may subsequently be made based on these preliminary flow calculations.

\footnotetext{
${ }^{*}$ Corresponding author at Trinity College Dublin.
}

E-mail address: bothaj@tcd.ie (J. Botha) 
Wind turbines generate noise from both mechanical and aerodynamic sources. Mechanical noise is produced by gearboxes, yaw mechanisms and other instruments required to convert wind power into electricity. Small turbines, however, have very few moving parts and very often incorporate direct drive generators in20 stead of gearboxes. The noise from these components is either very low or can easily be treated through the use of acoustic liners within the nacelle.

The primary noise generation mechanism present in modern SWT's is aeroacoustic noise [4. This noise source is produced by the aerodynamic effect of turbine blades interacting with incoming flow. The individual components of this noise source can be separated into a number of categories, namely: low-frequency noise, 25 inflow-turbulence noise, tonal noise and airfoil self-noise. Prediction of these noise sources, as applicable to turbine blades, is repeatedly accomplished by implementing codes [4 9] based on semi-empirical methods by; Brooks et al. 10] for airfoil self-noise, and analytic methods by Paterson and Amiet [11] for inflow-turbulence noise sources. These methods discretise a 3D turbine blade geometry into a number of $2 \mathrm{D}$ airfoil strip sections in space and then apply the models in a spanwise manner in time to account for blade rotation.

30 Low-frequency noise is not of major concern for wind turbines with an upwind configuration (blades ahead of tower) 12. Furthermore, the blade passing frequencies of modern VAWT's are of the order of several hertz, thus falling into the lower end of the audible noise spectrum - this noise source is probably not applicable to small turbines due to their lower relative operating speeds. Several studies have been performed to validate the aforementioned flow and noise prediction codes for their applicability to the problem of HAWT noise and continue to show good agreement with experiment [4, 13]. Such codes continue to be used in HAWT development.

VAWT blades undergo highly unsteady flow fluctuations as they rotate about their own axis [14, 15]. Unsteady forces act on these turbine blades due to geometric effects and blade-blade aerodynamic interactions; causing unsteady power generation with rotation. Unsteady flow will generate unsteady aeroacoustic noise 40 generation and the effect of these unsteady parameters on noise generation has not yet been explored in a prediction study.

Time dependent 2D and 3D Computational Fluid Dynamics (CFD) calculations have been seen to provide more detailed flow solutions for VAWT aerodynamics when compared to rudimentary stream tube models. CFD calculations can be used to quantify the anisotropy of turbulent fluctuations within the rotor. Fur-

45 thermore, wind tunnel testing of assembled VAWTs is difficult to perform and only a limited number of experimental results are available [16, 17. Experimental campaigns are mostly used to study overall performance as opposed to looking at detailed flow structures. Li et al. [16] performed a thorough investigation of the flow features around the blade of a VAWT, verifying CFD calculations against wind tunnel test data and showed that CFD calculations performed well when an appropriate turbulence model was applied. There are, 50 however, some outstanding issues to be addressed when using CFD for VAWT aerodynamic prediction [18. An example of this is the matter of grid dependency such as the study by Almohammadi et al. [19] showing the sensitivity of the power/torque calculations to mesh types and the time step/Courant number.

The primary objectives of the present study are concerned with the development of a validated computational method for the prediction of noise generated by a VAWT. Additionally the new methods will 55 make use of CFD calculations as input data to existing noise models since CFD calculations can be used to efficiently (and more accurately) predict detailed flow features including turbulence parameters which are important inputs required for noise prediction models. Models for inflow-turbulence and self-noise sources are implemented. This approach would realise the applicability of a hybrid noise prediction method for aerodynamically generated VAWT broadband noise sources. This study will aid in determining the location and mechanism of dominant noise sources arising from aerodynamically generated VAWT noise as well as understanding the effect that turbulence has on overall VAWT noise. The method is verified against an existing test case in order to establish its applicability to the present problem. Finally the method is used to study the effect of parameter variations on noise production for the SWIP V2 turbine, A 2kW VAWT developed by the consortium of partners working under the EU directive FP7 project, SWIP 1 .

\footnotetext{
${ }^{1}$ The overall objective of the SWIP project is to develop, implement and test innovative solutions and components for tackling the current barriers that wind turbines encounter, opening the way for a successful market uptake in the near future 20. This objective follows an EU directive to improve onshore wind power penetration with a goal of achieving $20 \%$ penetration by 2020 .
} 


\section{Airfoil Noise Models}

For this study, four noise prediction models were implemented. Two models were implemented to predict self-noise and another two for the prediction of inflow-turbulence noise. The results from each noise model will be compared in order to determine each ones' applicability to the prediction of VAWT noise.

A limitation of these noise models is that specific aerodynamic interaction between following blades is not considered - the blades are modelled in isolation. It is also assumed that the self-noise produced by the wind turbine blades is produced under steady flow conditions. In summary; the formulations assume steady, free stream, conditions under a quasi-steady time dependence.

\subsection{Airfoil Self-Noise}

Turbulent Boundary Layer (TBL) noise is produced by the scattering of the turbulent energy within a

75 hydrodynamic boundary layer by an airfoil trailing edge. The scattered energy propagates to the far field as an acoustic signal. Brooks et al. [10] modelled several types of airfoil self-noise in their paper. In this study blade tip effects are neglected. Quasi-tonal noise sources, such as those produced by vortex shedding and laminar boundary layer effects, generally do not manifest themselves in highly turbulent VAWT systems and are excluded from the modelling approach [21].

\section{i. Brooks Pope and Marcolini Model}

The commonly used airfoil self-noise model developed by Brooks et al. 10 (BPM model) relates the flow over an airfoil - in particular, the estimates of the boundary layer displacement thickness, to the radiated acoustic signal at the trailing edge. This model has been applied to noise predictions of other basic airfoils at a wide range of operating conditions (up to transonic speeds) with relatively good agreement, however, this model is limited by the fact that only a single airfoil type was considered 22.

The fundamental BPM model for TBL noise is defined by three equations with subscripts $p, s$, and $\alpha$ denoting the contribution to the overall airfoil noise for the pressure side, suction side and high angle of attack (separation-stall). These are

$$
\begin{gathered}
W_{p}(f)=10 \log _{10}\left(\frac{\delta_{p}^{*} M_{0}^{5} d D_{h}}{z^{2}}\right)+A\left(\frac{S t_{p}}{S t_{1}}\right)+\left(K_{1}-3\right)+\Delta K_{1} \\
W_{s}(f)=10 \log _{10}\left(\frac{\delta_{s}^{*} M_{0}^{5} d D_{h}}{z^{2}}\right)+A\left(\frac{S t_{s}}{S t_{1}}\right)+\left(K_{1}-3\right) \\
W_{\alpha}(f)= \begin{cases}10 \log _{10}\left(\frac{\delta_{s}^{*} M_{0}^{5} d D_{l}}{z^{2}}\right)+B\left(\frac{S t_{s}}{S t_{2}}\right)+K_{2} & \alpha<25^{\circ} \\
10 \log _{10}\left(\frac{\delta_{s}^{*} M_{0}^{5} d D_{l}}{z^{2}}\right)+A^{\prime}\left(\frac{S t_{s}}{S t_{2}}\right)+K_{2} & \alpha \geq 25^{\circ}\end{cases}
\end{gathered}
$$

and thus the sum of the contributions becomes a logarithmic sound pressure level addition,

$$
W_{\mathrm{BPM}}(f)=10 \log _{10}\left(10^{W_{p} / 10}+10^{W_{s} / 10}+10^{W_{\alpha} / 10}\right)
$$

where $W_{\mathrm{BPM}}(f)$ is the sound pressure level in third octave bands, $\delta^{*}$ is the boundary layer displacement thickness, $M_{0}$ is the inflow mach number, $d$ is the discretised blade span, $D$ is the directivity function, $z$ is the absolute distance to the receiver, the $S t$ variables are various definitions for Strouhal contributions described in the original NASA report, $A, A^{\prime}$ and $B$ are interpolation functions of experimentally derived curves and $K$ is an amplitude correction function - see [10. Eq. (3) switches at high angles of attack. When 95 the critical angle is reached $W_{p}=-\infty$ and $W_{s}=-\infty$. 


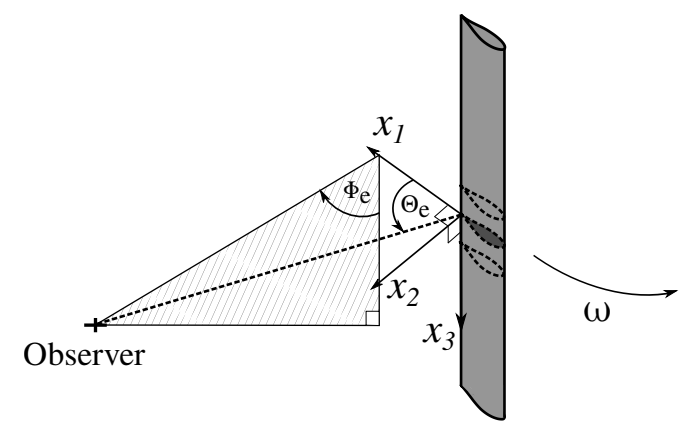

Figure 1: Local blade coordinate system and angles used for the directivity functions.

\section{ii. TNO models}

In order to account for the shortcomings of the BPM model and to improve noise prediction accuracy for non-symmetric airfoils in unsteady flow the TNO model was implemented. The TNO model for airfoil self-noise, developed by Blake and Parchen [23, 24, has gained much traction in the airfoil noise community of late and shows good agreement with measurements 25] 27. The version used in this study is known as the iTNO model [28] and reduces the number of input variables, decouples the convection velocity from the boundary layer height, and shows validated results that are in better agreement than previous studies.

The model incorporates a definition of the wavenumber frequency pressure spectrum which provides the changes in airfoil surface pressure at various wave numbers defined as,

$$
\Phi_{P}(\mathbf{k}, \omega)=4 \frac{\rho^{2}}{U_{c}} \int_{0}^{\infty} L_{t, 2}\left(x_{2}\right)\left(\frac{\partial U_{1}\left(x_{2}\right)}{\partial x_{2}}\right) \overline{u_{2}^{2}\left(x_{2}\right)} \phi_{22}\left(k_{1}\left(x_{2}, \omega\right)\right) e^{-2 x_{2}\left|k_{1}\left(x_{2}, \omega\right)\right|} \mathrm{d} x_{2}
$$

where $\Phi_{P}$ is the wavenumber frequency pressure spectrum as a function of $k$, the turbulent wavenumber, and $\omega$ the angular frequency where $\omega=2 \pi f$. The subscript of the wavenumber, $k$ denotes direction as per the coordinate system in Fig. 1. Other variables are defined in the previous section.

This equation is then closed with an expression for energy density spectrum, $\phi_{22}$ as a function of the wavenumber, $k$,

$$
\phi_{22}\left(k_{1}\right)=\frac{4}{9 \pi} \frac{\beta_{1} \beta_{3}}{k_{e}^{2}} \frac{\left(\beta_{1} k_{1} / k_{e}\right)^{2}}{\left[1+\left(\beta_{3} k_{1} / k_{e}\right)^{2}\right]^{7 / 3}}
$$

where $k_{e}$ is the wavenumber of the transverse integral length scale in the boundary layer defined as $k_{e}=0.7468 \sqrt{\pi} / 2 L_{t, 2} 29, \beta_{1}$ and $\beta_{2}$ are stretching factors defined by Lee 28 to be $\beta_{1}=1$ and $\beta_{3}=3 / 4$. The energy density spectra follows the form of a Von Karman turbulence spectrum that relates the incoming turbulence wavenumbers, $\mathbf{k}$, to the acoustic energy spectrum.

The farfield acoustic pressure spectral density (PSD), required to compare results to those of experiments is defined as a function of the angular frequency by,

$$
S_{p p}(\omega)=\left.\frac{d}{4 \pi z^{2}} \int_{-\infty}^{\infty} \frac{\omega}{c_{0} k_{1}} \Phi_{P}(\mathbf{k}, \omega)\right|_{k_{3}=0} d k_{1}
$$

where $d$ is discretised spanwise extent of the trailing edge, $z$ is the distance to the receiver, $c_{0}$ is the local speed of sound. The narrowband SPL is found from,

$$
W_{\mathrm{TNO}}(f)=10 \log _{10}\left(\frac{4 \pi S_{p p}(\omega)}{P_{\mathrm{ref}}^{2}} D\right)
$$

where $P_{\text {ref }}=20 \mu P a$, the factor $4 \pi$ is introduced to convert the double-sided spectrum to a single sided spectrum and convert back from angular frequency. $D$ is the directivity function defined later. 


\subsection{Inflow-Turbulence Noise}

Inflow-turbulence noise is a source of aerodynamic noise produced as airfoil blades interact with inflow turbulence. Amiet, and then later Paterson and Amiet [11, 29], define expressions for the far-field acoustic power spectral density of an airfoil in a turbulent stream. Amiet defines incoming turbulence using the Von Karman spectrum and this model is seen to be well suited in its applicability to aircraft operating in highly are equivalent at large length scales encountered by large wind turbines. One outcome of this study will be to determine whether these models are applicable to small VAWT noise prediction.

\section{i. Paterson and Amiet's Inflow-Turbulence Noise Model}

The model of Paterson and Amiet presented 11 is presented here in SI units noting that some unit components of noise are combined using a blending function as proposed by Lowson [30] and Moriarty and Migliore [31.

The model is defined by the following equations,

$$
\begin{gathered}
W_{\text {Inflow }}=W_{\text {Inflow }}^{H}+10 \log _{10}\left(\frac{\mathrm{LFC}}{1+\mathrm{LFC}}\right) \\
W_{\text {Inflow }}^{H}=10 \log _{10}\left[\frac{\rho_{0}^{2} c_{0}^{2} d}{2 z^{2}} M_{0}^{3} \overline{u^{\prime 2}} L_{t} \frac{\left(k / k_{e}\right)^{3}}{\left(1+\left(k / k_{e}\right)^{2}\right)^{7 / 3}} D\right]+78.4 \\
\mathrm{LFC}=10 S^{2}\left(1+9 \alpha^{2}\right) M_{0}\left(\frac{k c}{2}\right)^{2} \beta^{-2} \\
S^{2}=\left[\frac{2 \pi k c}{2 \beta^{2}}+\left(1+2.4 \frac{k c}{2 \beta^{2}}\right)^{-1}\right]^{-1}
\end{gathered}
$$

where $\rho_{0}$ is the density of the medium, $c_{0}$ is the speed of sound, $D$ is the directivity function, $I_{t}$ is turbulent intensity, $k$ is the wavenumber $\left(k=(2 \pi f) / U_{0}\right), L_{t}$ is the inflow turbulent length scale, $d$ is local airfoil span width, $c$ is chord length, $M$ is the mach number, LFC is a low frequency correction, $\alpha$ is the local airfoil angle of attack, $S$ is an approximation of the compressible Sears function [30] and $\beta^{2}=1-M^{2}$.

\section{ii. Buck's Inflow Noise Model}

Buck et al. 13] proposed a modification to the Paterson and Amiet model for calculating inflow-turbulence noise. He suggested applying the Kolmogorov turbulence spectrum 32] in place of the Von Karman spectrum as it was seen that, for large turbines, at sufficiently high frequencies and large enough atmospheric length scales the models converged to the same result. This modification makes it easier to measure turbulence parameters experienced by the turbine as it is easier to measure turbulent dissipation than a turbulent length scale. Furthermore the new method requires shorter measurement times which further speeds up predictions. By replacing the turbulence spectrum with that of Kolmogorov, the turbulence parameter of interest then becomes, $\epsilon_{t}$ the turbulent dissipation rate $\left(\mathrm{m}^{2} / \mathrm{s}^{3}\right)$. Buck's model modifies Eq. 10 to obtain,

$$
W_{\text {Inflow }, B}^{H}=10 \log _{10}\left[\frac{\rho_{0}^{2} c_{0}^{2} d}{2 z^{2}} M_{0}^{3} \epsilon^{2 / 3} k^{-5 / 3} D\right]+77.6
$$

where the rest of the model is used in the same manner as in Eqs. (9) - (12). The results from both models will be presented in this paper. 


\subsection{Directivity}

Due to the relative motion between the airfoil and the stationary receiver, of the order of Mach 0.1 , a doppler amplification factor is introduced [33]. This directivity factor accounts for retarded coordinates of source emissions.

If noise producing eddies are sufficiently small compared to convection velocities i.e. acoustic wavelengths are shorter than airfoil chord lengths then the directivity function follows the formulation of Brooks and observer is,

$$
G_{\mathrm{BB}, j}(f)=\sum_{b=1}^{s_{n}} \sum_{d=1}^{\mathrm{ts}} \frac{n}{\mathrm{n}_{\phi}}\left(\frac{f}{f_{0}}\right)_{b d}\left[G_{j}(f)\right]_{b d}
$$

where $G_{\mathrm{BB}}$ is the overall acoustic pressure signal for a given noise model, the subscript $j$ refers to one of the four noise models discussed above. $n$ is a weighting factor applied for the number of blades, $n_{\phi}$ is shift, a correction factor to account for time compression and expansion effects due to the relative blade flow in the freestream [34]. $[G(f)]_{b d}$ represents the acoustic pressure at the observer location for a given noise model, $j$.

\section{Noise Prediction Methodology}

185

The methodology used to predict VAWT noise is essentially similar to that of prior codes used for HAWT noise prediction [37] but is, however, reformulated for the vertical rotation of the turbine blades. A single blade of the specified turbine geometry is discretised in 3D space into a series of strips, each representing an airfoil chord of finite span. Fig. 2 shows a representative turbine blade discretised into four spanwise 


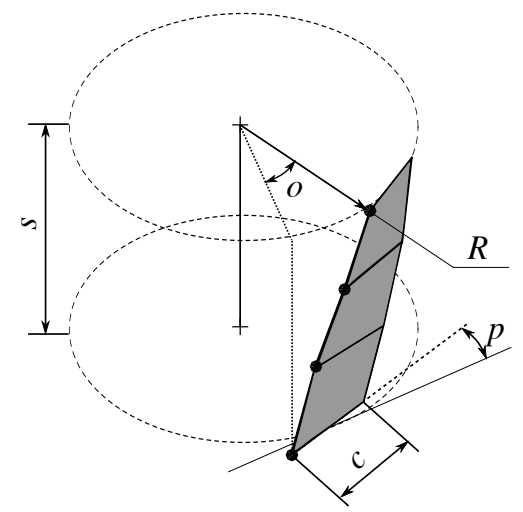

Figure 2: Geometric parameters required to model a turbine blade. indicates trailing edge.

sources with all geometric parameters required to define the machine. The approach also further discretises the domain in time allowing the blade to advance along its rotational path for a single revolution. As a result the computational domain is split into a discrete number of spanwise and radial grid points.

The turbine's operating conditions, rotational velocity (RPM) as well as the wind speed are required to define the simulation. The wind speed, also known as the domain inlet velocity, corresponds to the required atmospheric air speed needed for the device to rotate at the given RPM. Additional parameter required are

195 the time step and number of blade sources, necessary to define the discretisation as well as the location of the listener. The required inputs are listed in Table 1.

\begin{tabular}{llll} 
Category & Parameter & Symbol & Units \\
\hline Geometry & Radius & $R$ & $\mathrm{~m}$ \\
& Blade Span & $s$ & $\mathrm{~m}$ \\
& Blade Chord & $c$ & $\mathrm{~m}$ \\
& Blade Pitch & $p$ & $\circ$ \\
& Helical Twist & $o$ & $\circ$ \\
& Number of Blades & $n$ & \\
Operating Conditions & Rotational Velocity & $\omega$ & $\mathrm{rad} / \mathrm{s}$ \\
& Inlet Velocity & $U_{\infty}$ & $\mathrm{m} / \mathrm{s}$ \\
Turbulent Conditions & Turbulent Intensity & $I_{t}$ & $\%$ \\
& Integral Length Scale & $L_{t}$ & $\mathrm{~m}$ \\
Simulation Parameters & Angular Discretisation & $n_{\phi}$ & \\
& Sources Along Span & $s_{n}$ & \\
& Receiver Location & $\mathbf{z}_{\mathbf{i}}$ & $\mathrm{m}$
\end{tabular}

Table 1: Input parameters required to define a simulation.

Once all simulation parameters are defined, the aerodynamic model can be solved. The aerodynamic model describes the flow over the blade, either at the blade inlet or along the airfoil trailing edge boundary layer depending on which model is being evaluated. A flow solution is required to calculate noise using the aforementioned prediction models. Table 2 describes the flow input parameters required for each of the given noise models. The density of the medium, $\rho_{0}$, is also required for calculation and is assumed constant.

The flow input parameters, described in Table 2, are typically extracted from experimental data and used to tune models 10, 13, 34. However, when performing noise predictions for new rotor designs these data are often not available until late in the project's timeline. Analytical predictions of the required input parameters can be performed to support measured data in early project development.

In this study two approaches were used to calculate the flow input parameters of Table 2. The first approach used analytical models which calculate velocity and angle of attack from geometric assumptions and thereafter solve for the turbulence using a combination of fundamental approaches primarily based on 


\begin{tabular}{llll} 
Noise Source & Model & Flow Inputs & Notes \\
\hline Self Noise & BPM & $\delta_{i}^{*}, U_{0}, \alpha$ & \\
& TNO & $L_{t, i}, U_{i}, k_{t}$ & In the boundary layer \\
Inflow-Turbulence & Paterson & $L_{t}, I_{t}, U_{0}, \alpha$ & At blade inlet \\
& Buck & $\epsilon, U_{0}, \alpha$ &
\end{tabular}

Table 2: Flow inputs required to solve each noise model.

idealised flat plate theories or semi-empirical models. The second approach made use of pre-calculated 2D

\section{Analytical Flow Parameter Modelling}

This section describes the models used to analytically predict inflow and boundary layer flow parameters. Equations for flow modelling are presented, or derived, and relationships between flow parameters and turbulence are discussed. These are all related to the problem of airfoil aerodynamics as applied to a rotating VAWT coordinate system.

\subsection{Blade Inflow Velocity and Angle of Attack}

Blade inflow velocity and angle of attack are reconstructed from geometric relations of velocity described by vectors of flow in Fig. 3a, where $\lambda=(\omega R) /\left(U_{\infty}\right)$ is the tip speed ratio 38. Assuming the 2D blade section rotates at a constant velocity and encounters a constant freestream velocity; the local blade angle of attack and inlet velocity can be calculated from geometric vector addition,

$$
\begin{aligned}
& \alpha=\tan ^{-1}\left(\frac{\sin \Phi}{\cos \Phi+\lambda}\right)+p \\
& U_{0}=U_{\infty} \sqrt{1+2 \lambda \cos \Phi+\lambda^{2}}
\end{aligned}
$$

where $\alpha$ is the effective local blade angle of attack, $\Phi$ is the azimuthal angle as defined in Fig. 3, $p$ is the fixed pitch angle of the airfoil, $U_{\infty}$ is the domain inlet velocity and $U_{0}$ is the local inflow velocity at the blade inlet, essentially the freestream velocity equivalent for a $2 \mathrm{D}$ infinite airfoil in flow. The local blade inlet velocity, $U_{0}$, is one of the most important parameters for noise prediction and accurate prediction thereof is essential.

\subsection{Turbulence Parameters}

The noise prediction models used in this study require several turbulence parameters at the local blade inlet in order to relate the flow solution to the acoustic signal radiated by the airfoil section. Models for inflow-turbulence noise require two parameters, the integral length scale of turbulence, $L_{t}$, and the turbulence intensity, $I_{t}$, or alternatively the turbulent dissipation rate, $\epsilon$. For HAWT noise prediction these parameters are generally measured at the turbine site prior to installation and noise analysis.

\section{Integral Length Scale}

The integral length scale of turbulence at the airfoil inlet is defined as a convenient measure of the extent of the region over which turbulent velocities are appreciably correlated [32. As a physical quantity it describes

the size of the largest energy bearing eddies in a turbulent flow. Using the assumption of isotropic turbulence, this length scale is defined as,

$$
L_{t, 1}\left(x_{1}\right)=\int_{0}^{\infty} R_{11}\left(x_{1}\right) \mathrm{d} x
$$

where $R_{11}\left(x_{1}\right)$ is the axial cross-correlation of the turbulent velocity in the flow direction, $u_{1}^{\prime}, L_{t}$ refers to the integral length scale with the subscript denoting the directional extent of the quantity with coordinates defined as per Fig. 1. When using the analytical model, the inlet turbulence parameters remain constant with rotation. Therefore the correct average measure of this parameter within the rotor is important. 


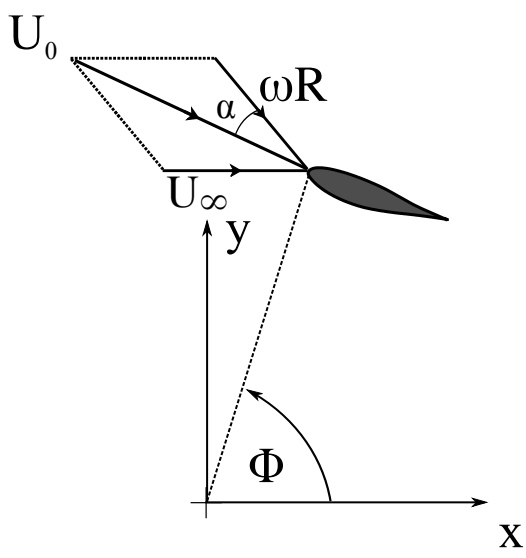

(a)

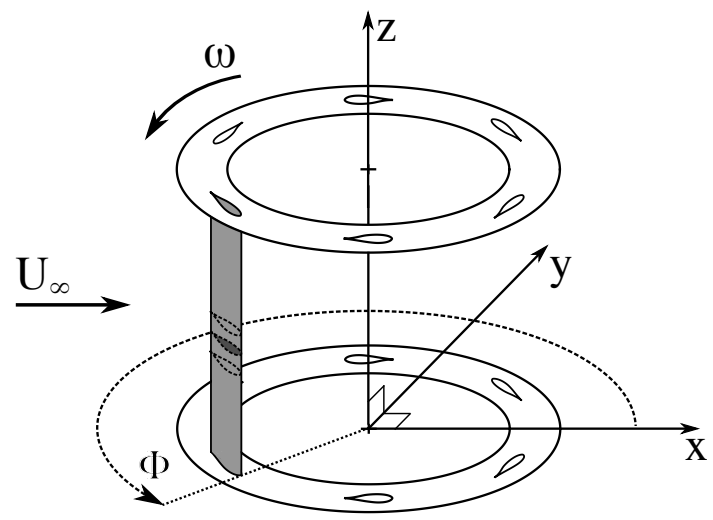

(b)

Figure 3: Coordinate system used throughout this paper. (a) 2D Local blade coordinates and flow relationships. $U_{\infty}$ is inflow velocity, $\omega$ is rotational velocity, $R$ is radius, and $\alpha$ is defined as the effective angle of attack of the airfoil. Azimuthal angle, $\Phi$, is defined as zero on the $\mathrm{x}$ axis and increases positively with counterclockwise rotation. (b) 3D coordinate system showing the location of inlet velocity in relation to rotating airfoil blades.

\section{Transverse Integral Length Scale in the Boundary Layer}

The length scale measured in the boundary layer as used as input to the TNO noise model differs to that of the inflow integral length scale of Eq. (19). Whilst the more commonly used length scale is referred to as the longitudinal integral correlation scale; the length scale considered here is related to the vertical dimensions of turbulent eddies - a distinction made clear by Kamruzzaman et al. 39. The definition thereof is the integral of the normalised spatial two point correlation coefficient of the vertical velocity fluctuations. We refer to this length scale as the transverse length scale, denoted by a subscript 2 to indicate the direction,

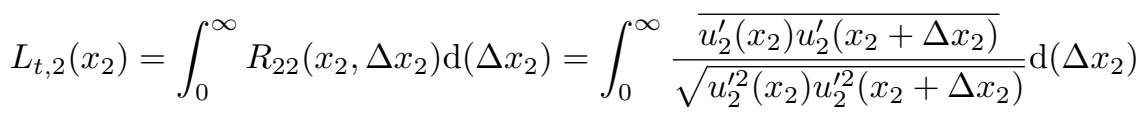

where $R_{22}$ is the correlation coefficient between the turbulent velocity $\overline{u^{\prime}}$ normal to the airfoil surface and the same signal separated by $\Delta x_{2}$. This definition of integral length scale is the same as in Eq. 19 however the direction of the flow parameters has been altered and the location is no longer in the freestream but along the airfoil trailing edge. This length scale can be solved for analytically by starting with Prandtl's mixing length hypothesis to solve for the mixing length, $l_{m}$,

$$
l_{m}=0.085 \delta \tanh \left(\frac{\kappa x_{2}}{0.085 \delta}\right)
$$

where $\kappa$ is the Von Karman constant, $\kappa=0.41$, and $\delta$ is the boundary layer thickness defined as $\delta=$ $0.99 U_{\infty}$ at the airfoil trailing egde. The above relationship is non-physical as the mixing length does not

255 increase linearly through the boundary layer. A damping function is applied near the wall by means of the Klebanoff damping function as proposed by Kamruzzaman et al. [40,

$$
l_{m}=\frac{0.085 \delta \tanh \left(\frac{\kappa x_{2}}{0.085 \delta}\right)}{\sqrt{1+5.5\left(\frac{x_{2}}{\delta}\right)^{6}}}
$$

and then by definition the mixing length is related to the transverse integral length scale of turbulence,

$$
L_{t, 2}=\frac{l_{m}\left(x_{2}\right)}{\kappa}
$$




\section{Turbulent Kinetic Energy}

Turbulent kinetic energy describes the kinetic energy per unit mass within the turbulent velocity fluctu-

\section{Turbulent Dissipation Rate}

Turbulence dissipation, $\epsilon$, is the rate at which turbulence kinetic energy, $k_{t}$ is converted into thermal internal energy. The quantity is a necessary input parameter to the inflow-turbulence noise model of Buck 13 and is directly calculated using CFD. However, for analytical modelling the turbulent dissipation rate

$$
\epsilon=c_{\epsilon} \frac{k_{t}^{3 / 2}}{L_{t, 1}}
$$

Where $c_{\epsilon}=0.55$ is a constant selected to match the high frequency asymptote of the Buck model to that of the Paterson and Amiet model for inflow-turbulence noise predicted using the analytic approach. 


\subsection{Analytic Boundary Layer Parameters}

The TNO model 28] required reconstructed velocity and turbulence boundary layer profiles as input to noise prediction models. The general approach in literature is to use XFoil 43 to solve for; the freestream velocity, $U_{0}$; wall skin friction coefficient, $C_{f}$; boundary layer displacement thickness, $\delta^{*}$; and the boundary layer momentum thickness, $\theta$. However, for this analysis non-iterative analytic models for flat plates in flow were implemented for simplicity.

\section{Skin Friction Coefficient}

Skin friction is a qualitative measure of the ratio between wall shear stress and dynamic pressure for a fluid bounded by a wall. A switching function is used in order to estimate the skin friction coefficient, $C_{f}$, of an airfoil by approximating it as a flat plate with a transition Reynolds number of 500000 using the following equations from Blasius and Schlicting respectively 44,

$$
C_{f}=\left\{\begin{array}{cc}
1.328 / \sqrt{\operatorname{Re}} & \operatorname{Re}<500,000 \\
0.73 / \log (\operatorname{Re})^{2.58} & \operatorname{Re} \geq 500,000
\end{array}\right.
$$

where Re is the Reynolds number defined as,

$$
\operatorname{Re}=\frac{\rho_{0} U_{0} c}{\mu}
$$
quantified errors between experimental and analytically determined acoustic results.

\subsection{Test Case}

The quietrevolution QR5 turbine was selected as a verification case for the analytical prediction method. extensive experimental work performed in measuring the turbine's noise output [46. The primary geometric parameters of the turbine are given in Table 3. Certain parameters related to the design of the turbine are unknown; the blade pitch angle, $p$,is assumed to be zero, the helical angle of the blades as it appears from figures is 90 degrees. The turbine radius is non-constant with height, having a slight egg-beater taper design, to rotate at a relatively constant tip speed ratio of 3.5 which would correspond to the optimum efficiency point for this configuration.

Reported measurements [46] were carried out using the BS EN 61400 standard for wind turbine noise measurements on a full scale machine installed in a relatively open field. Measurements were taken at night 320 to reduce background noise from a nearby highway. Corrections for background noise were applied using

where $\kappa=0.41$ is the Karman constant, $u^{*}=U_{0} \sqrt{C_{f} / 2}, U_{w}$ is the wake velocity defined as, $U_{w}=1-\cos \left(\pi x_{2} / \delta\right)$ and $B=5.5$ is a constant [45].

\section{Verification of the Analytic Flow Model}

In order to confirm that the noise models have been implemented correctly and to determine the accuracy of the predictions when using the analytical flow model, a verification study was performed. This approach 


\begin{tabular}{ll} 
Parameter & Value \\
\hline Chord Length & $0.175 \mathrm{~m}$ \\
Blade Pitch & $0^{\circ}$ \\
Radius & $1.5 \mathrm{~m}$ \\
Span & $5 \mathrm{~m}$ \\
Helical Twist & $90^{\circ}$ \\
Airfoil & NACA 0018 \\
Number of blades & 3 \\
Tower height & $18.5 \mathrm{~m}$ \\
TSR & 3.5
\end{tabular}

Table 3: Parameters defining the geometry of the QR5.

the given standard by subtracting mean background noise from the turbine's noise signal and correcting for values where the difference between the levels was less than $3 \mathrm{~dB}$.

Four microphones were used, each located at a $22.5 \mathrm{~m}$ radius away from the turbine on the ground. Two of the microphones were located directly upstream and downstream of the turbine whilst two additional microphones were located along the radius downstream at an angle of $45^{\circ}$ to the flow direction.

Measurements were taken for several wind speeds from 6-10 m/s, each of which corresponded to increases in rotor RPM but maintained a relatively constant operating TSR (Tip Speed Ratio). Time, wind speed and direction were recorded at one minute intervals and used to determine the one-third octave band spectra and A-weighted noise levels. Measurement uncertainty in the one-third octave band spectra was deemed to be 330 typically $1.7 \mathrm{~dB}$.

Tonal content of the noise was determined from narrowband spectra using A-weighted measurements, and possible tones were identified when the local maximum of the frequency content was more than $6 \mathrm{~dB}$ above the average noise level. The results showed slight possible tones existing from $20 \mathrm{~Hz}-120 \mathrm{~Hz}$.

Turbulence statistics were not measured on site during these tests. However, following computational analysis by Pearson 21 it was found that there exists a series of turbulent length scale and intensity parameter pairs that produce the same spectral levels when applying the inflow-turbulence noise model of Paterson and Amiet [1]. This approach was used to work back from the known solution to find a set of working turbulence parameters. Making the assumption of a wake turbulence of at least $15 \%$ the model of Pearson was tuned to find a working turbulent length scale that was roughly equal to the airfoil chord length. For perspective, 340 the atmospheric length scales experienced by large wind turbines are normally of the order of $\tilde{1} 0-100 \mathrm{~m}$. These length scales can be determined using atmospheric boundary layer standards such as IEC 61400-1 that extrapolate known velocity and estimated surface roughness measurements to solve for the turbulent length scale and intensity parameters at hub height.

Setting the turbulence length scale to such low values concentrates all the turbulent energy into much smaller length scales than those in the atmosphere and can, possibly, lead to non-physical results. However, based on CFD analysis for the later cases in this study, it was seen that the length scales experienced by the VAWT blades were indeed much lower than the level of the freestream atmospheric values. Thus, for this particular analysis the length scale, as experienced by the rotating blades, was set to the chord length $(0.175$ $\mathrm{m})$ and the turbulence intensity set to $15 \%$.

\subsection{Validation of the Airfoil Self-Noise Models}

Using the current approach, the TNO self-noise model was validated against single airfoil results to determine its applicability to the current problem. The result in Fig. 4 compares results from the BPM and iTNO self-noise models to experimental results from Brooks et al. 10. Measurements were taken for a NACA 0012 airfoil a meter overhead of the trailing edge. Compared to Lee 28] the implementation of the 355 iTNO model in this study slightly overpredicts the peak frequency whilst underpredicting high frequency components when compared to measurements. One key difference between the two implementations is the use of different aerodynamic solvers - Lee [28 making use of XFoil to determine boundary layer parameters. Using the existing approach, the iTNO model provides similar results to that of the traditional BPM model and as a result is at the least comparable and applicable to wind turbine noise predictions. 




Figure 4: Validation of the self-noise models, $\alpha=0, U_{\infty}=39.6 \mathrm{~m} / \mathrm{s}, \times$ $\mathrm{TNO},+\mathrm{BPM}, \bullet$ Experiment

\subsection{Sound Pressure Level Predictions}

Predictions were performed for the QR5 at integer wind speeds from $6-10 \mathrm{~m} / \mathrm{s}$, the length scale was set to a constant $0.175 \mathrm{~m}$ and the turbulent intensity to $15 \%$. Unweighted simulation results are presented in Fig. 5 for all four noise models. The results show SPL in third-octave bands as compared to the measurements of Dyne [46. Results show a consistent trend of increases in overall noise levels at all frequencies as inflow velocity is increased.

From these figures the dominant noise source is inflow-turbulence noise. Across the frequency range this noise source dominates over self-noise by about $20 \mathrm{~dB}$. This dominance by inflow-turbulence noise is consistent with previous predictions and experiments [21, 47]. The predicted inflow-turbulence spectra for both the Buck and Paterson models converge at frequencies beyond $100 \mathrm{~Hz}$, emphasising that the Buck model is applicable to the current problem. Furthermore, inflow-turbulence noise spectra largely follow the trend of the measured data in that the levels increase with increases in inflow wind speed, particularly at higher wind speeds where the mid-frequency spectral hump begins to dissipate.

\section{Airfoil Self-Noise Model Analysis}

The self-noise models employed in the analysis are not consistent with each other and predict different frequency peaks. The BPM model predicts a peak noise frequency at about $100 \mathrm{~Hz}$ whilst the TNO model predicted peak is at about $2000 \mathrm{~Hz}$. The peak frequency for the BPM model is attributed to stall noise which occurs during VAWT operation when the airfoil goes past the critical angle and flow separation onsets. This noise source is characteristed as increases in low frequency contributions to airfoil self-noise. Stall noise contributions are not predicted in the TNO model.

The peak frequency predicted by the TNO model is directly proportional to the maximum value of $\Phi_{22}$ in Eq. (6) which is a function of the transverse integral length scale of turbulence [28. A lower maximum transverse length scale in the boundary layer leads to a higher peak frequency. The analytic transverse length scale prediction, as described in Eqs (21) - (22), is a function of the boundary layer displacement thickness and that in turn is a function of Reynolds number and angle of attack. Fig. 6 shows the maximum value of the turbulent length scale in the boundary layer with changes in azimuthal angle as predicted by the analytic model. The peak shifts more to the right as this maximum value increases. The pressure side contributions maintain long periods of low maximum values meaning that the spectral peak will be most affected by these values. In this analysis the peak frequency for the TNO model predictions falls in the range of about 2000 $3000 \mathrm{~Hz}$ and is attributed to increased pressure side noise contributions due to the reduced boundary layer thickness. 




(a)



(c)



(b)

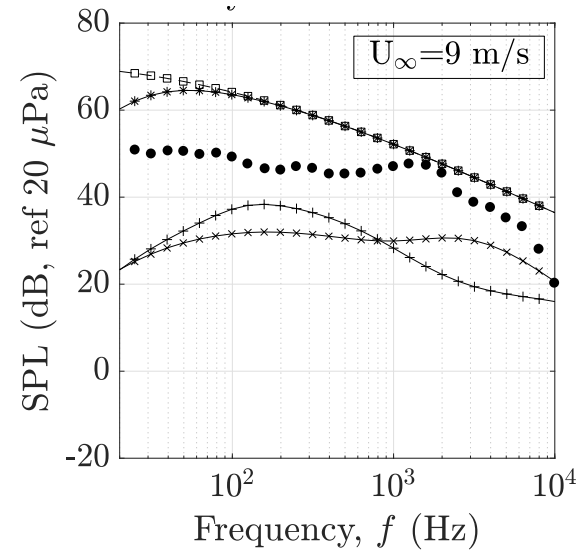

(d)



(e)

Figure 5: Comparison between experiments and predictions for the QR5 wind turbine using the analytical approach with changes in wind speed indicated in the figures; + TBL - BPM; $\times$ TBL - iTNO; $*$ Inflow - Paterson and Amiet; $\square$ Inflow - Buck; $\triangle$ Inflow prediction - Pearson; $\nabla$ TBL prediction - Pearson; • Measurements [4].

\subsection{Overall Sound Level Trends}

In Fig. 7 A-weighted OASPL measurements are compared to analytic predictions at several integer wind speeds. OASPL was calculated by adding contributions of TBL and inflow-turbulence noise together. Since 


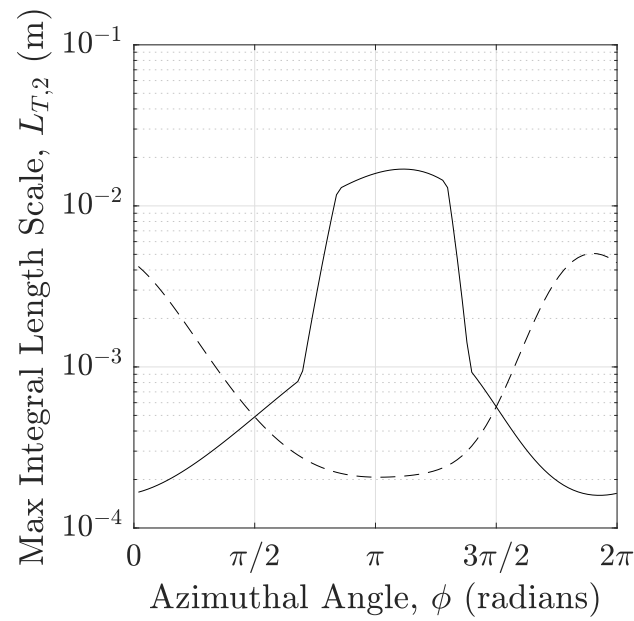

Figure 6: Maximum value of $L_{T, 2}(x)$ in the boundary layer with azimuth for the QR5 turbine at a wind speed $U_{\infty}=10 \mathrm{~m} / \mathrm{s},---$ suction side and, --- pressure side.

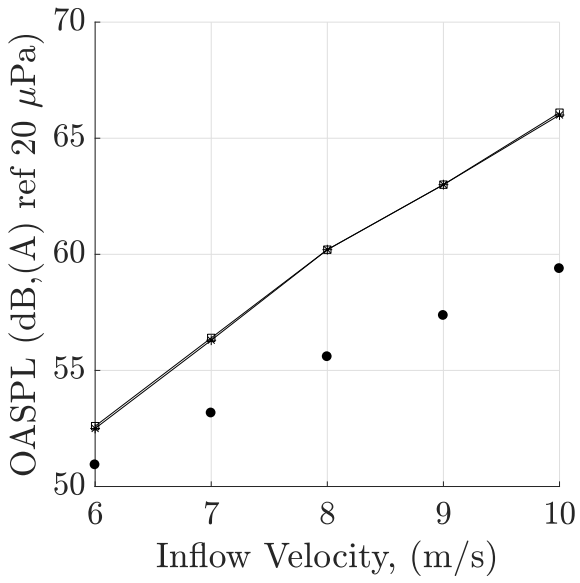

Figure 7: A-weighted OASPL levels comparing, $\bullet$ measured data, $\square$ Buck, $\times$ Paterson.

inflow-turbulence noise was dominant, addition of either of the TBL noise model prediction results yielded negligible increases in the result. The figure shows the results of the Buck and Paterson models nearly superimposed on top of each other.

Measurements show a linear increase in OASPL with increases in integer wind speeds. There is an overprediction by the model that is emphasised at higher wind speeds. At a speed of $8 \mathrm{~m} / \mathrm{s}$ the gradient of the graph changes. This change in slope is captured in the model, however, the overall slope differs between results.

\section{Comparisons to Pearson's Predictions}

Fig. 5e shows results of the predictions of this study compared to predictions made by Pearson [21. Pearson used the BPM model for calculating airfoil self-noise and the Amiet model for inflow-turbulence noise. In addition to these models the aerodynamic solution required for input parameters was calculated using an experimental approach which resolved blade velocities from acoustic measurement techniques.

Compared to the results of Pearson, self-noise noise largely follows the same trend as the predictions made using the TNO method. Inflow-turbulence noise predictions, however, are overpredicted by a factor of about $10-15 \mathrm{~dB}$ across the. The seemingly accurate predictions of Pearson are possibly due to the use an incorrect form of the inflow-turbulence model purported by Lowson [30] which propagates an error in the use 
410 of CGS units used for the density and speed of sound terms resulting in a prediction 10 - 20 dB lower than models implementing correct unit conventions. This result verifies that computations for TBL noise have been performed with sufficient accuracy when compared to existing models and quantifies the error in the existing approach for calculating inflow-turbulence noise.

\section{CFD Modelling and Data Extraction}

415

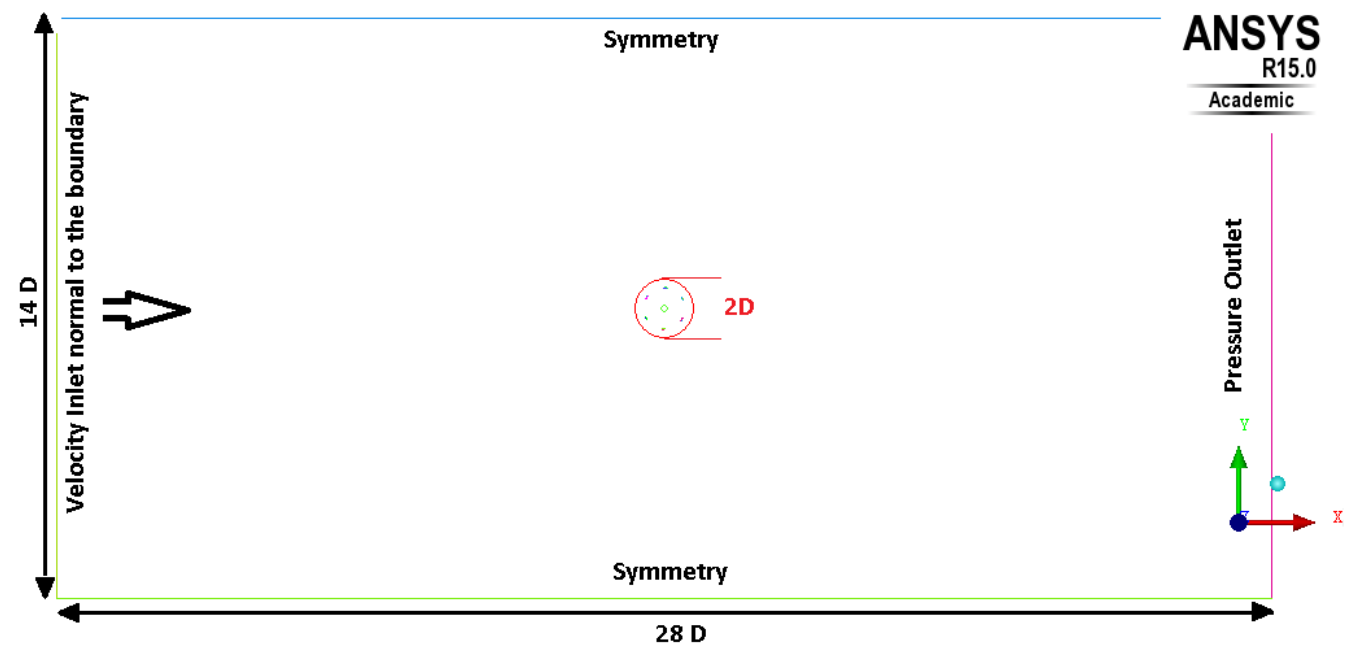

Figure 8: Computational domain dimensions and boundary conditions. D refers to the rotor diameter it the centre of the domain.

The SST k- $\omega$ turbulence model is a two-equation eddy-viscosity model which uses the shear stress transport (SST) formulation and is the most appropriate model forVAWT flow simulations due to its effective treatment of all floww conditions near a wall [50. The model also performs well when predicting adverse pressure gradients necessary to model flow separation [51.

A VAWT produces large amounts of unsteadiness between passing blades. To ensure numerical convergence and accurate flow results a number of modelling procedures have been followed.Ma et al. [49] shows that a wide range $30<y+<200$ can predict the flow accurately. For this case the value of $y+$ at the airfoil wall is of order of less than 10. From flat plate equations it is estimated that the boundary layer thickness, ${ }_{435} \delta$, will be about $0.54 \mathrm{~mm}$ and thus the first cell distance from wall is set to have a maximum value $0.2 \mathrm{~mm}$ to ensure an acceptable $y+$.

For VAWT simulations a Courant Friedrichs Lewy (CFL) value of the order of 40 is shown to provide accurate flow results [19. Due to the implicit time integration implemented, there is no need to use a very small CFL number. However, smaller CFL values will provide more stability to the solution. For this case the CFL number is less than 10 throughout key regions in the domain. The selected time step was determined 
to fulfil the CFL criteria. Several time steps were tested which corresponded to an azimuthal rotation of between $0.24^{\circ} /$ step and $0.8^{\circ} /$ step. A time step of $0.72^{\circ} /$ step was selected as the final result. The domain was also allowed to complete 13 revolutions before convergence was determined and results obtained.

\subsection{Data Extraction}

Velocity and turbulence parameters are exported from the CFD calculations at each distinct time step, for a single rotation of the rotating CFD domain. The data probe extracts meaningful flow parameters at three locations around the airfoil as seen in Fig. 9 . Fig. 9 is extracted from the CFD calculation (described later) highlighting the airfoil nomenclature used. This figure also shows the results of considering the airfoil as existing in a stationary (black arrows) and rotating reference frame (blue dashed arrows). The approach is described in Eq. (38).

The exported data files are collected into a folder which can be read by the prediction method. When the code is running, the azimuthal angle of the blade segment currently being evaluated is converted to a numeric value pertaining to a specific time step file and the file corresponding to the particular spatial location is read into the code accordingly.

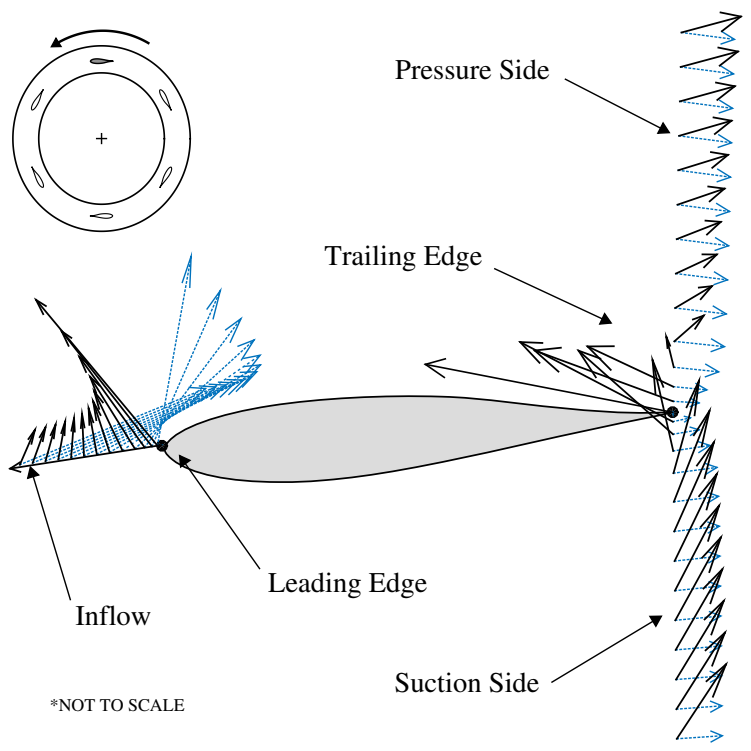

Figure 9: Extraction probes and velocity vectors at LE and TE of airfoil in the stationary CFD coordinate system - and the rotating reference frame - - -. The number of velocity vectors have been reduced for clarity.

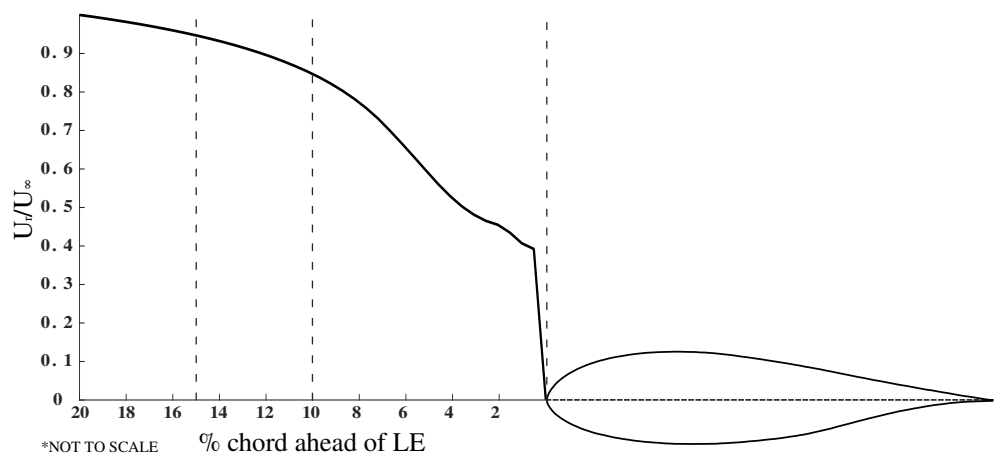

Figure 10: Velocity ahead of the airfoil leading edge non-dimensionalised with effective freestream velocity 


\subsubsection{Blade Inflow Velocity and Angle of Attack}

For the CFD analysis; angle of attack and blade velocity are calculated using the extracted data. The extracted velocity vector is in a body fixed coordinate system and needs transformed to a coordinate system fixed at the centre of rotation of the turbine to account for the rotational speed of the turbine. In order to obtain the local blade inflow velocity, $U_{0}$, the velocity at the leading edge needs to be averaged to reduce the effect of the zero relative velocity at the stagnation point at the airfoil leading edge (Fig. 10). The local blade inflow velocity is found by integration over the leading edge probe between selected locations from,

$$
\mathbf{U}_{\mathbf{0}}=\frac{1}{m-n} \int_{n}^{m}\left(\mathbf{U}_{\mathbf{0}, \mathbf{L E}}+\omega \mathbf{R}\right) \mathrm{dLE}
$$

where $\mathbf{U}_{\mathbf{0 , L E}}$ is the vector of local blade inflow velocity extracted from the leading edge probe, $\omega$ is the angular velocity with respect to the centre of rotation of the turbine, $\mathbf{R}$ is the distance from a point to the turbine radius, and $n$ and $m$ are the range of locations ahead of the leading edge defined as a percentage of

465 the chord as seen in Fig. 10. Angle of attack is defined as the angle between the inflow velocity and a vector from the chord leading edge to trailing edge. This is calculated as,

$$
\alpha=\left\{\begin{array}{cc}
\operatorname{atan} 2\left(U_{0, x 2} / U_{0, x 1}\right)-\operatorname{atan} 2\left(c_{x 2} / c_{x 1}\right) & |\alpha|<\pi / 2 \\
\arccos \left(\mathbf{c} \cdot \mathbf{U}_{\mathbf{0}}\right) /|\mathbf{c}|\left|\mathbf{U}_{\mathbf{0}}\right| & |\alpha| \geq \pi / 2
\end{array}\right.
$$

where $U_{0, i}$ is the local blade inflow velocity and $c$ is the blade chord, atan2 is defined as the four quadrant inverse tangent [52. $x 1$ and $x 2$ denote blade coordinates as per Fig. 1 .

\subsubsection{Tuning the Inflow Model}

Using the above approach the angle of attack and blade inflow velocity are calculated for a reference case and compared to the geometric solution. The results seen in Fig. 11] show strong sensitivity to the use of data within the first $10 \%$ of the probe (nearest to the wall) and a much lower sensitivity when moving the probe further ahead of the leading edge. Fig. 11a shows good visual agreement for the blade inflow velocity using probes capturing 10-20\% and 15-20\% of data ahead of the leading edge. The correct velocity trend 475 is captured, however, a much lower maximum velocity is observed when compared to the analytic solution which can physically attributed to confluent effects between following blades. The angle of attack as seen in Fig. $11 \mathrm{~b}$ shows much better agreement than velocity in that both the maximum and minimum values of angle of attack for the probes capturing $10-20 \%$ and $15-20 \%$ of data ahead of the leading edge are comparable to the maximum and minimum of the geometric solution (Eq. 17) within a few degrees. In both of these results

480 convergence occurs after moving the probe location to either $10-20 \%$ or $15-20 \%$ a convergence as localised stagnation point effects are removed. The range selected for all calculations was the $15-20 \%$ range. Thus, in Eq. (34), $n=0.15$ and $m=0.2$.

At an azimuthal angle of $3 \pi / 2$ there is a sudden change in angle of attack due to dynamic stall of blades 53. During stall the flow over the airfoil separates and, from an acoustic perspective, the blade begins to

${ }_{485}$ act as a bluff body in flow. For light stall conditions, airfoil noise generally increases in intensity by up to 5 $\mathrm{dB}$ and reduces the peak frequency [54].

\subsection{Turbulence Parameters}

By performing CFD calculations, a better local prediction of the turbulent parameters ahead of an airfoil blade can be considered. The exact definition of these parameters will change depending on the computational 490 turbulence model used. In this study the k- $\omega$ SST model [41] was considered. Using this model several turbulence parameters become directly available namely the turbulent kinetic energy, $k_{t}$; turbulence intensity, $I_{t}$; turbulent dissipation rate, $\epsilon$; and, specific dissipation rate, $\omega_{t}$. Several relationships need to be provided to calculate the missing parameters.

\section{Integral Length Scale}

495 Since the integral length scale of turbulence is an unsteady flow parameter it needs to be redefined as a statistical quantity based on available data. This is performed by making use of a definition from the k- $\omega$ SST turbulence model,

$$
L_{t, 1}=\frac{\sqrt{k_{t}}}{\omega_{t} c_{\mu}}
$$




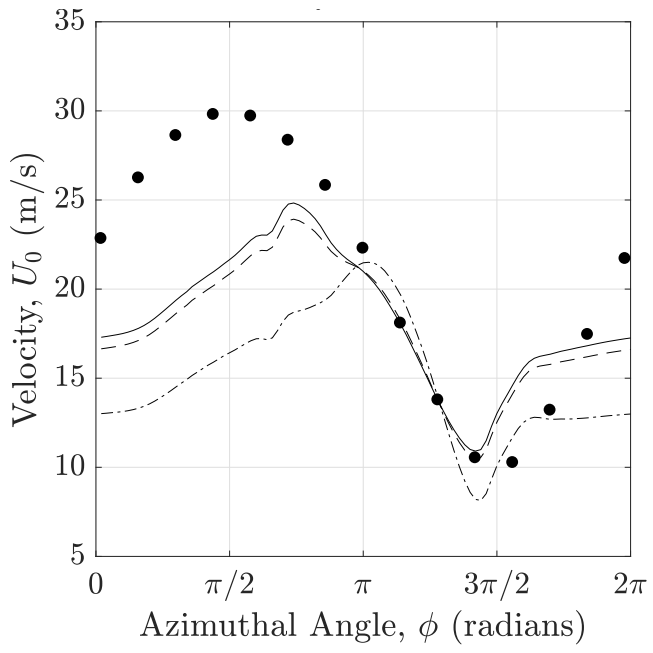

(a)



(b)

Figure 11: Key flow parameters with changes in azimuth showing the effect of the amount of data sampled ahead of LE with azimuth. (a) Local blade inlet velocity, (b) angle of attack. $-15-20 \%,--10-20 \%,-\cdot-0-20 \%$,

- Geometric solution (Eq. $17 \cdot 18)$ ).

where $\omega_{t}$ is the specific dissipation rate of turbulence and $c_{\mu}=0.09$ is a constant as defined by Menter [41. Thus, expressions are derived to calculate the integral length scale, Eq. (36), and intensity, Eq. (26),

of turbulence based on outputs of the k- $\omega$ SST turbulence model.

\section{Transverse Integral Length Scale in the Boundary Layer}

Assuming homogeneous, isotropic turbulence the longitudinal and transverse length scales can be related [55, 56] as $L_{t, 1}=2 L_{t, 2}$. By using the assumption of isotropic turbulence, this means that the transverse length scale can now be calculated from CFD data by combining this relation with Eq. (36),

$$
L_{t, 2}=\frac{1}{2} \frac{\sqrt{k_{t}}}{\omega_{t} c_{\mu}}
$$

Kinetic Energy in the Boundary Layer

The quantity of kinetic energy in the boundary layer, $k_{t}$, can be extracted directly from the pressure and suction side boundary layer probes.

\section{Boundary Layer Reconstruction from CFD Informed Method}

The trailing edge boundary layer velocity profile can be extracted from the CFD results by making use of the velocity and position data along the trailing edge extraction probes. These profiles are used to calculate the boundary layer thickness, $\delta$, and the displacement thickness, $\delta^{*}$. Velocity at the trailing edge needs to be normalised in a direction parallel to the airfoil chord line. This process is defined by a number of flow relationships and computational operations described below.

\section{i. Read Data and Interpolate}

For any given numerical time step, the CFD data file corresponding to that location is read into MATLAB. Input data consists of the velocity, $\mathbf{U}_{\mathbf{i}, \mathbf{C F}}$, and position, $\mathbf{x}_{\mathbf{i}}$, vectors along the probe, where the subscript $i$ denotes the cartesian coordinate either $x$ or $y$. Each vector is interpolated onto a linearly spaced grid of a reasonable size for the required computational accuracy using the MATLAB pchip interpolation method to perform a shape-preserving piecewise cubic interpolation. 
ii. Calculate Velocity Profile

The input velocities from the CFD calculation do not consider the moving reference frame. The rotational velocity is added to the stationary velocity by,

$$
\mathbf{U}_{\mathbf{i}}=\mathbf{U}_{\mathbf{i}, \mathbf{C F D}}+\omega\left(\mathbf{x}_{\mathbf{i}}-\mathbf{x}_{\mathbf{c}}\right)
$$

where $U_{i}$ is the velocity at any given location in the boundary layer, $\omega$ is the rotational velocity of the turbine, and $x$ is a nodal location. Subscript $i$ represents a cartesian coordinate along the boundary layer, subscript CFD represents the velocity extracted
cartesian coordinate of the centre of the turbine.

Once the rotational velocity has been included, the magnitude of the velocity vectors is calculated and the scalar projection of the velocity parallel to the chord is taken,

$$
\mathbf{U}_{\mathbf{B L}}=\left|\sqrt{\mathbf{U}_{\mathbf{x}}^{2}+\mathbf{U}_{\mathbf{y}}^{2}}\right| \cos \theta
$$

where $\theta$ is the angle between the velocity vector, $\mathbf{U}_{\mathbf{i}}$, and the airfoil chord calculated using atan2, the chord line at the trailing edge.

\section{iii. Boundary Layer Detection}

The arbitrary assignment of the boundary layer thickness, $\delta$, is generally defined as the location where a bounded flow reaches $99 \%$ of the freestream flow velocity, $\delta=0.99 U_{\text {infty }}$. Due to blade-blade aerodynamic 535 interactions flow across the trailing edge is normally not 'well behaved' and thus a new definition for the boundary layer thickness is proposed for this study as,

$$
\delta=0.95 \max \left(\mathbf{U}_{\mathbf{B L}}\right)
$$

The MATLAB findpeaks function is used to determine where local maxima in the boundary layer velocity profile occur. The maxima are sorted and a value of $95 \%$ of this maxima is selected as the corresponding boundary layer thickness value. If this function fails (i.e. the local maxima lies at the end of the data) then $95 \%$ of the global maximum value is used as the boundary layer thickness value.

The boundary layer displacement thickness, $\delta^{*}$, is defined as,

$$
\delta^{*}=\int_{0}^{\infty}\left(1-\frac{\mathbf{U}_{\mathbf{B L}}\left(x_{2}\right)}{\max \left(\mathbf{U}_{\mathbf{B L}}\right)}\right) \mathrm{d} x_{2}
$$

where the coordinate $x_{2}$ is defined as being perpendicular to the airfoil chord (see Fig. 1).

Fig. 12 shows a series of non-dimensionalised boundary layer velocity profiles extracted from a CFD case compared to the solution to Eq. (33) for the given azimuthal angles seen in the figure title. The CFD profiles 545 shows some deviation from the analytic solution which is to be expected due to blade confluence. In general, for most angles in the range, the shape of the profile is captured accurately and consistently.

\section{CFD Based Prediction Results}

Using input data from the CFD calculations noise predictions for an unique six-bladed VAWT were performed. Several parameter studies were conducted leveraging the enhanced detail afforded by the com550 putations over that of the analytical predictions.

\subsection{Case Comparison}

The SWIP consortium has proposed a six-bladed $2 \mathrm{~kW}$ vertical axis wind turbine, dubbed the SWIP V2, designed for an unique roof mounted application. A 3D schematic of the blades is seen in Fig. 13 and the specifications of the turbine are summarised in Table 4. The turbine produces a peak power output of 2

$555 \mathrm{~kW}$ which corresponds to an operating tip speed ratio of around 2.0. This operating point as well as several other cases relevant to the turbine design are considered and summarised in Table 5. Cases 1-3 have varying 



(a)
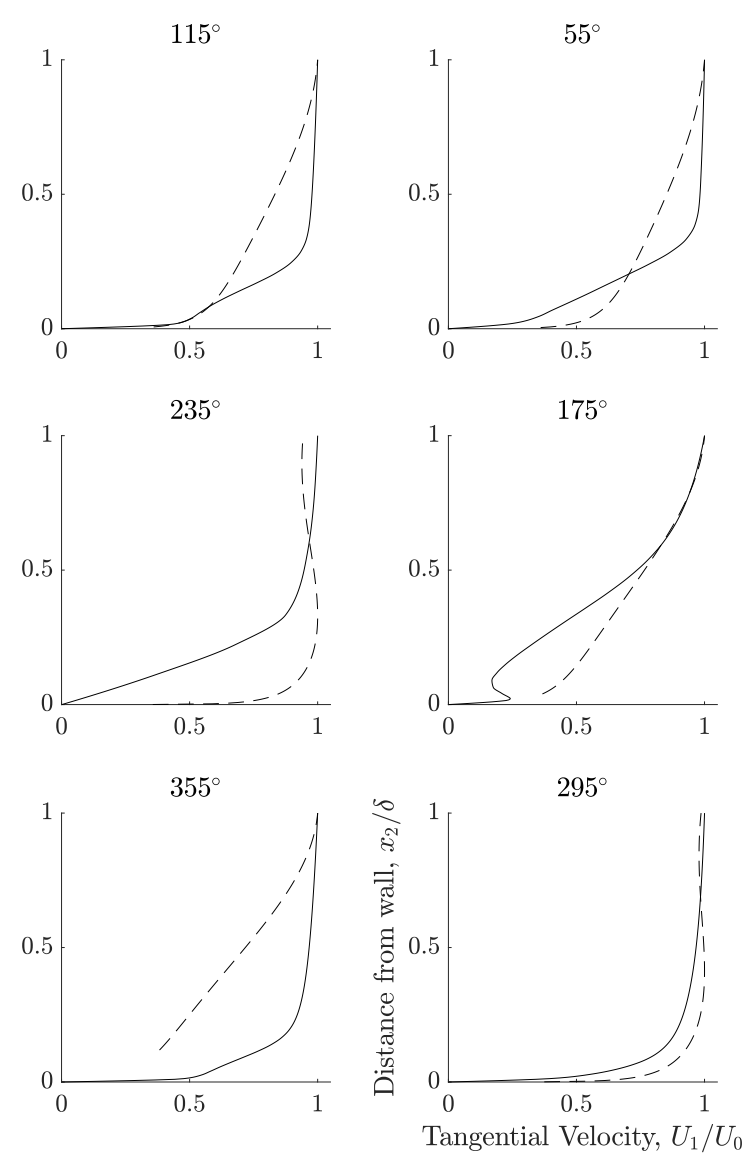

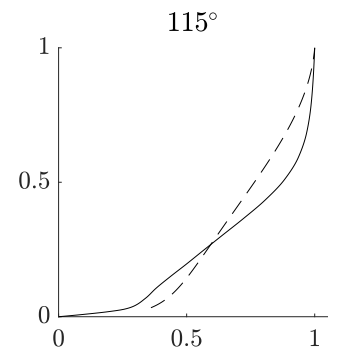

$235^{\circ}$


Figure 12: Non-dimensionalised boundary layer velocity profiles for azimuthal angles $\phi$. (a) Suction side (b) Pressure side. - CFD Data, - Coles' Law of the wall/wake.

tip speed ratios as governed by changes in inlet velocity and RPM values. Cases 4 and 5 are dynamically equivalent to case 2 but have different levels of inflow turbulence.

Turbulence intensity and length scale values used as boundary conditions at the computational domain inlet were derived from measurements taken at the test site according to the IEC61400-1 standard for definitions of atmospheric length scale measurements. These parameters, detailed in Table 5 are prescribed as freestream conditions at the computational domain inlet. As the turbulence levels experienced within the rotor do not match those in the freestream [57, 58, CFD is used to provide a more accurate representation of the levels of turbulence experienced by the blades themselves which will differ to the freestream conditions.

\subsection{Convergence and Grid Independence}

The prediction method makes use of large data banks CFD data. In order to predict noise it is not necessary to sample every available data point. A grid consisting of 5 sources along the span and 25 radial locations was sufficient to obtained a converged solution.

Since CFD simulation results are sensitive to increases in mesh density, a grid refinement study was

performed to determine the effect of these refinements on the results of the acoustic study. The computational grid used for the CFD calculations was refined by increasing the number of cells in the mesh by about 70\%, as well as increasing the number of local grid points around each airfoil by the same percentage. Results of the grid sensitivity study are presented in Table 6. The table shows the OASPL for each of the four noise models using two separate CFD grids. OASPL was calculated on the ground 1.5 hub heights away from the 


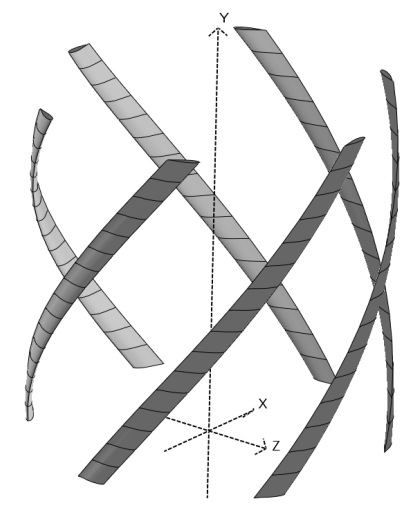

Figure 13: 3D model of the SWIP V2 vertical axis wind turbine blades.

\begin{tabular}{ll} 
Parameter & Value \\
\hline Chord Length & $0.183 \mathrm{~m}$ \\
Pitch & $0^{\circ}$ \\
Radius & $1 \mathrm{~m}$ \\
Span & $2 \mathrm{~m}$ \\
Helical Twist & $90^{\circ}$ \\
Number of blades & 6 \\
Airfoil & DU06-W-200 \\
Tower height & $10 \mathrm{~m}$
\end{tabular}

Table 4: V2 turbine parameters defining the geometry of the VAWT. The helical twist is defined as an azimuthal rotation of the blade along span in the rotor coordinate system.

\begin{tabular}{lllllll} 
Case & $U_{\infty}(\mathbf{m} / \mathbf{s})$ & $\mathbf{R P M}$ & $\mathbf{T S R}$ & $L_{t}(\mathbf{m})$ & $I_{t}(\mathbf{\%})$ & $\mathbf{C p}(\mathbf{C F D})$ \\
\hline 1 & 8.4 & 200 & 2.49 & 7 & 20 & 0.136 \\
2 & 10 & 190.1 & 1.99 & 7 & 20 & 0.398 \\
3 & 12 & 169.2 & 1.48 & 7 & 20 & 0.267 \\
& & & & & & \\
4 & 10 & 190.1 & 1.99 & 7 & 10 & 0.398 \\
5 & 10 & 190.1 & 1.99 & 7 & 5 & 0.398
\end{tabular}

Table 5: Test cases for current study. Each case represents operating conditions on the power curve. Turbulence parameters are measured freestream conditions from meteorological measurements on site. The power coefficient (Cp) was determined from the CFD analysis.

575 turbine downstream. The models all show a negligible change in OASPL with changes in cell count. This result emphasises that the models are relatively insensitive to increases in mesh density.

\subsection{Dominant Noise Sources}

The QR5 turbine was dominated by inflow-turbulence noise produced by interactions between turbulence in the rotor and the blades themselves. When compared to experiments this noise source was overpredicted.

580 In this section CFD data from the computational campaign was extracted and used as input data for the noise prediction models in order to compare noise predictions between the two approaches. This analysis considers case 2 from Table 5 .

Fig. 14 presents a comparison of these noise predictions using both the analytical prediction method as well as the CFD based method. In this result the CFD based method shows lower levels of inflow-turbulence noise when compared to the analytical approach. This is quantified as an overall reduction in SPL at all 


\begin{tabular}{llll} 
& Grid 1 & Grid 2 & Difference \\
\hline Mesh Cells & 1500000 & 2600000 & $73 \%$ \\
Airfoil Grid Points & 300 & 520 & $73 \%$ \\
& & & \\
BPM & $40.5 \mathrm{~dB}$ & $41.5 \mathrm{~dB}$ & $2.5 \%$ \\
TNO & $34.9 \mathrm{~dB}$ & $35.1 \mathrm{~dB}$ & $0.6 \%$ \\
Paterson & $62.8 \mathrm{~dB}$ & $61.5 \mathrm{~dB}$ & $2.1 \%$ \\
Buck & $67.5 \mathrm{~dB}$ & $67.0 \mathrm{~dB}$ & $0.7 \%$
\end{tabular}

Table 6: Effect of mesh refinement on OASL predictions

frequencies of the order of $15 \mathrm{~dB}$, and this deviation can be attributed to a more rigorous prediction of local blade inflow velocity and turbulence intensity at the local blade inlets. Fig. 11a compares the blade inlet velocity with azimuth for the analytical and CFD based approaches of case 2, and shows that CFD based results deviate considerably from the geometric solution with a peak velocity prediction roughly $5 \mathrm{~m} / \mathrm{s}$ lower than the analytic baseline. This discrepancy in the two approaches leads to large scale differences in the SPL predictions due to the power dependence of the Mach number term Eq. 10.



(a)

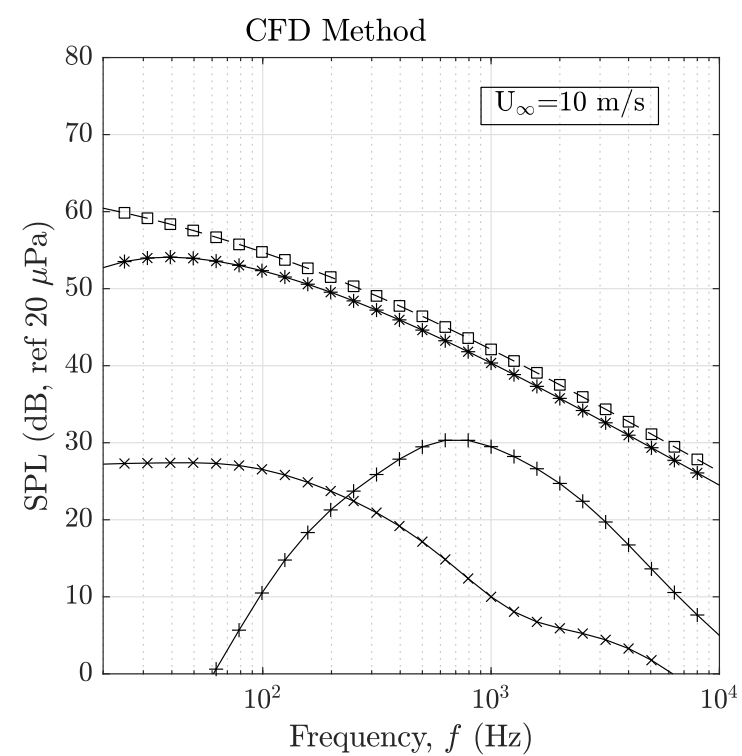

(b)

Figure 14: Prediction of self-noise and inflow-turbulence noise using the (a) analytical and (b) CFD method; + TBL - BPM; $\times$ TBL - iTNO; * Inflow - Paterson and Amiet; $\square$ Inflow - Buck

The analytical prediction of self-noise is comparable to previous predictions with similar trends observed for the intensity ans spectral peak. Results from the TNO model show a shifted peak frequency. The CFD based prediction method yields different results to the analytical approach. BPM predicted noise from this broad low frequency trend but rather a very distinct peak frequency at $1000 \mathrm{~Hz}$ with the rest of the signal rolling off around this location.

Using CFD data to inform the TNO model reduces the overall level of predicted noise by about 10 $\mathrm{dB}$. In the verification study, the analytical approach yielded a slight overprediction in noise compared to experimental results. The current result shows a general reduction in noise predicted at all frequencies as well as shift in the peak noise frequency concentrating more of the signal in the lower frequency region.

The analytical flow model approach utilises a series of empirical equations to solve for the airfoil boundary layer thickness and other input parameters. By using CFD calculations to inform the noise models, the aero- 
dynamic model is solved in the time domain. This has implications for noise prediction in that, even though 605 the noise model is applied using a quasi-steady assumption, the aerodynamic flow model is not. This has the implication that more of the problem is now considered from a time domain perspective thus alleviating the problem of a steady flow assumption used for the analytical flow modelling approach. Furthermore, the TNO method, with its more accurate physical descriptions of noise based on the entire boundary layer profile, is able to provide improved accuracy for the current problem.

\subsection{Noise Source Localisation}

Due to unsteady aerodynamic effects, noise emitted by a VAWT is not constant throughout rotation. It is possible to determine at which azimuthal location the source strength will be at a maximum. This was accomplished by setting the value of the directivity function to be a constant of 1 for a single airfoil rotation. Furthermore three dimensional effects are also neglected. By extracting the value of all noise sources ${ }_{615}$ throughout a single rotation a plot is produced that shows the source strength distribution for the turbine with azimuthal rotation (Fig. 15) for CFD case 2. The plot shows the predicted frequency content of noise for both self-noise and inflow-turbulence noise sources as the turbine blade undergoes a single rotation. Both plots are scaled equally and are presented in third-octave bands. From these plots, the location of maximum noise is at an azimuthal location of $\pi$. This corresponds to the location directly upwind of the turbine.

There is a reduction in the level of source noise observed at an azimuthal location of $3 \pi / 2$ corresponding to the location where dynamic stall occurs. Local blade inflow velocity rapidly reduces to a value of less than zero over a comparably brief angular range. This sudden reduction in velocity accounts for a reduction of noise of about $20 \mathrm{~dB}$ in Fig. 15b. When looking at the source localisation contour for TBL noise, a peak is observed near the azimuthal location of $3 \pi / 2$. In this case high frequency noise reduces to lower levels whilst the low frequency components appear to remain high. A distinct shift in the peak frequency is observed in the region between $\pi$ and $3 \pi / 2$. Physically this is ascribed to an increase in the boundary layer turbulence in this region.

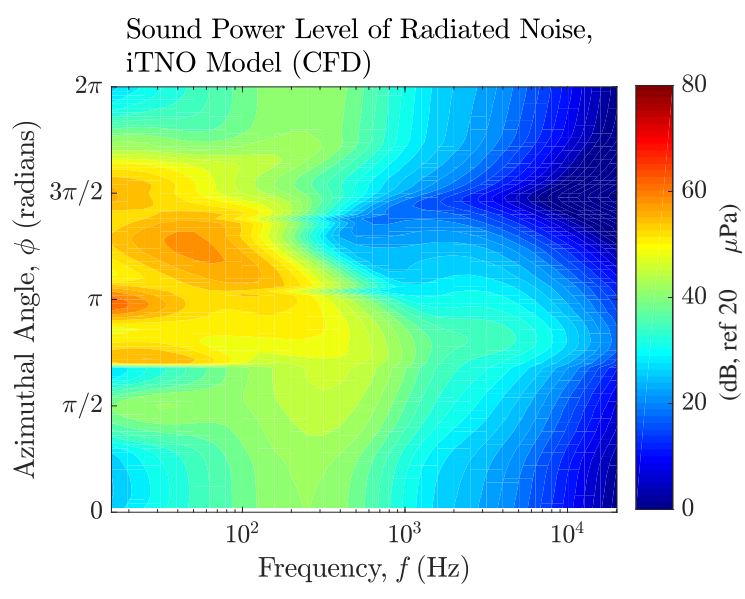

(a)

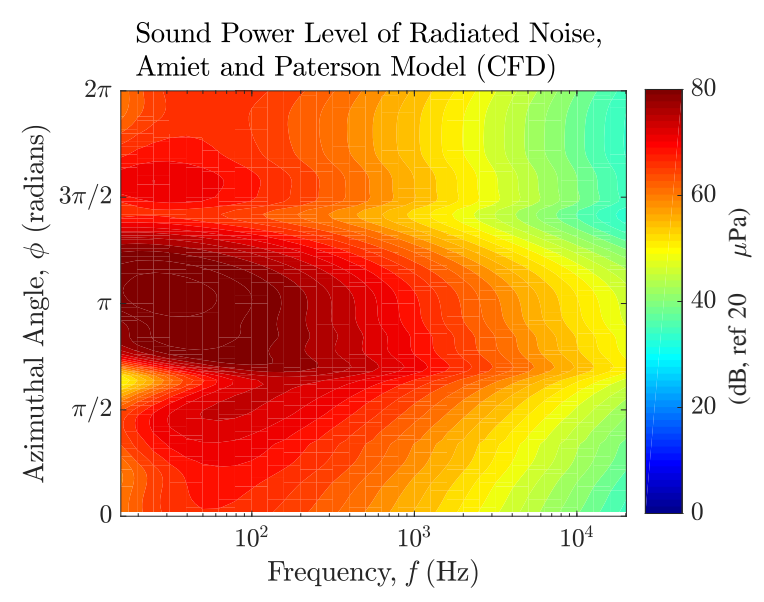

(b)

Figure 15: Spectra showing frequency content of noise, predicted using (a) iTNO and (b) Paterson and Amiet model, with change in azimuthal angle.

\subsection{Directivity}

VAWT noise has a slight directional component to it [46. Provided that wind direction is consistent, this directional effect will be observable as close as one hub height away from the turbine and is quantified as a change in the OASPL levels observed at various azimuthal locations around the device. Case 2 is considered for this analysis.

Fig. 16 shows the change in OASPL level with azimuth for listeners located around the turbine at 1.5 hub heights away. This plot shows similar trends to Fig. 15b. The Paterson and Amiet model for inflow-

turbulence noise is considered. The source strength plot (Fig. 16a) shows the value of the OASPL at the 
source excluding the directivity factors of Eqs. 114) and (15). The loudest source region occurs at a location aligned with the maximum blade velocity (Fig. 11a). When considering directivity corrections, this source noise region gets considerably attenuated due to the effect of low frequency airfoil noise directivity consistent with those used in the calculation of inflow-turbulence noise [13, 59].

For the OASPL associated with directivity, source strength is a function of azimuth with the loudest region occurring close to $2 \pi$. Source strength is amplified by the distance to the receiver where the loudest region is that one where the highest velocity occurs closest to the receiver, thus reducing the distance to the receiver, $z$, and increasing local noise. The inclusion of the directivity factors (Fig. 16b leads to a reduction in the level of noise observed at the receiver location. Attenuation is a factor of the blade geometry in relation to the receiver. These directivity factors are geometrically a function of azimuthal blade position with respect to listener location as well as being dynamically linked to an inverse proportionality factor of the blade velocity. These interlinked factors make for directivity plots that are not symmetric around the wind axis with the loudest region occurring downstream of the turbine.

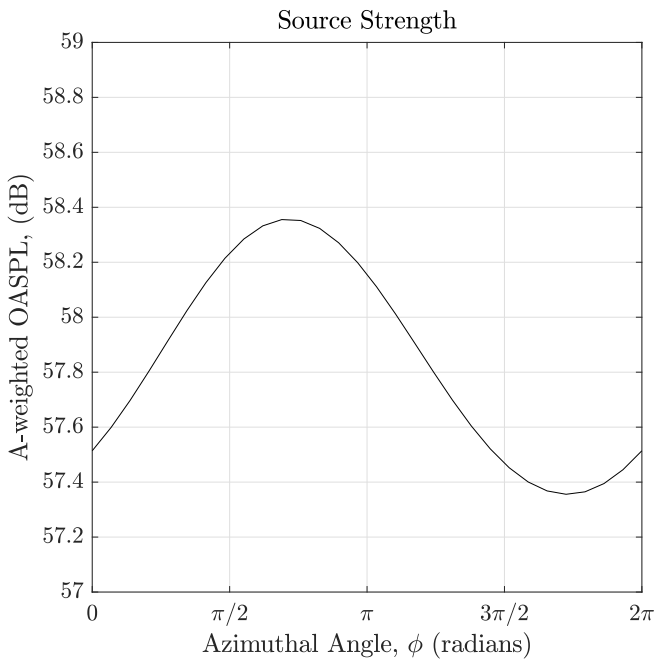

(a)

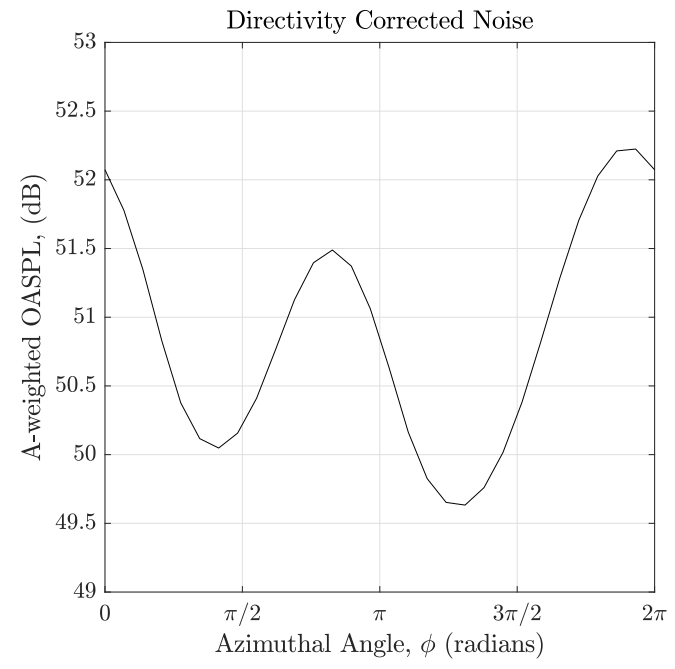

(b)

Figure 16: OASPL at various azimuthal locations on ground level one hub height away from the turbine (a) without and (b) with directivity corrections applied.

\subsection{Effect of Varying Flow Conditions}

The domain inlet velocity and turbine RPM were adjusted to determine the effect of changes in operating conditions on overall noise production. Cases 1-3 from Table 5 were used for this analysis. As the domain inlet velocity is increased so too is the maximum angle of attack and local blade inlet velocity with azimuth. Fig. 17 shows these parameters with changes in azimuthal angle for the range of varying inflow conditions. As the boundary conditions change so to does the maximum velocity within the rotor. Cases 1 and 2 experience very similar maximum velocities (Fig. $17 \mathrm{a}$ ) with a difference of about $1 \mathrm{~m} / \mathrm{s}$ observed at roughly the same azimuthal angle whilst case 3, however, shows a large increase in the maximum velocity which will directly affect noise levels. For the case of $12 \mathrm{~m} / \mathrm{s}$ inlet velocity, the dynamic stall event, characterised by a very sudden angle of attack change seen in Fig. 17b, is much more dramatic.

Turbulence levels within the rotor generally increase with increases in domain inflow velocity. There is a clear trend of increasing turbulent velocity as inflow velocity increases (Fig. 18b). Turbulent length scales, on average, also increase with increasing inflow velocity (Fig. 18a)

Fig. 19 shows the overall noise prediction using the Paterson and Amiet model for inflow-turbulence noise for the three cases. The listener is located at a standard location, on the ground 1.5 hub heights downstream of the turbine. Increasing the domain inlet velocity directly increases the overall noise produced. This increase is as a result of changes seen in the dynamic and turbulence parameters within the rotor. 




(a)

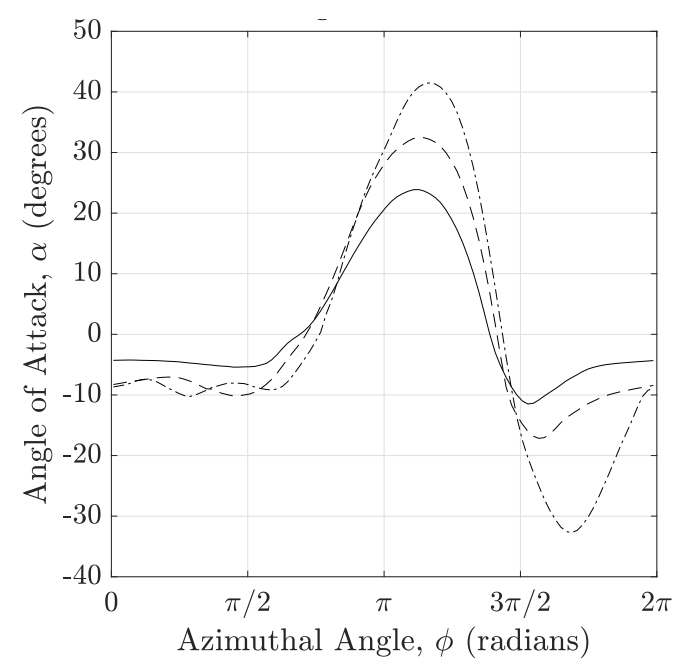

(b)

Figure 17: Flow parameters at local blade inlet for changes in domain inlet velocity $\left(U_{\infty}\right)$ (a) local blade inlet velocity (b) angle of attack with azimuth; $-8.4 \mathrm{~m} / \mathrm{s},--10 \mathrm{~m} / \mathrm{s},-\cdot-12 \mathrm{~m} / \mathrm{s}$



(a)

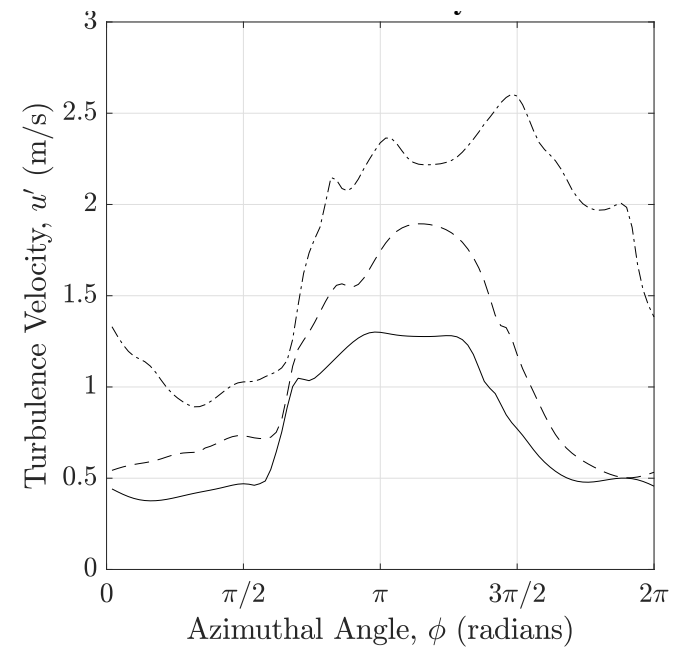

(b)

Figure 18: Turbulence parameters at local blade inlet for changes in domain inlet velocity $\left(U_{\infty}\right)$ (a) turbulent length scale (b) turbulent velocity with azimuth; - $8.4 \mathrm{~m} / \mathrm{s},--10 \mathrm{~m} / \mathrm{s},-\cdot-12 \mathrm{~m} / \mathrm{s}$

\subsection{Effect of Turbulence}

CFD computations have the added benefit of being able to easily compute the levels of turbulence within a rotor. For VAWT's operating in urban and peri-urban environments high levels of turbulence are to be expected and can affect the level of inflow-turbulence noise - a noise source which increases in proportion to the amount of turbulence in the system. The Paterson and Amiet model in Eq. (10) scales to the square of the mean turbulent velocity, meaning that a doubling of turbulent velocity leads to an increase in isolated airfoil noise of $6 \mathrm{~dB}$. For this analysis the turbulence intensity at the computational domain inlet was modified to determine the effect of this change on overall noise levels. CFD cases 2, 4 and 5 from Table 5 were considered for this section, these cases all have the same dynamic boundary conditions bar modifications to the domain inlet turbulence intensity. 


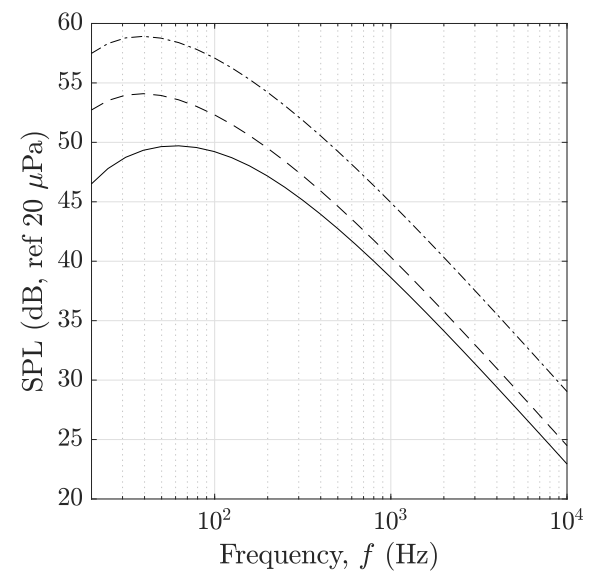

Figure 19: SPL with changes in domain inflow velocity $\left(U_{\infty}\right)$ using the Paterson and Amiet model with CFD input data; $-8.4 \mathrm{~m} / \mathrm{s},--10 \mathrm{~m} / \mathrm{s}$, $-\cdot-12 \mathrm{~m} / \mathrm{s}$

\section{Rotor Turbulence}

Fig. 21d shows local blade inlet velocity within the rotor for several different inlet turbulence parameters. As the domain inlet turbulence intensity increases, the aerodynamic system is not appreciably affected emphasising that any changes in observed noise spectra are due to modified turbulence parameters. As inlet turbulence intensity increases so to do the overall levels of turbulence experienced within the rotor. Fig. 21 a shows that the turbulence velocity increases appreciably from inlet turbulence of 5 to $10 \%$ and that an increase to $20 \%$ turbulence intensity does not have such a drastic increase on the overall levels.

Fig. 20 shows the level of turbulent kinetic energy within the domain of the rotor, the quantity of which is proportional to the turbulent velocity, $\overline{u^{\prime}}$ seen in Fig. 21a. The development of the wake region in both cases is complete at a location of $4 \mathrm{D}$ downstream of the rotor. Fig. 20a shows a case where inflow turbulence is set at $5 \%$. For this case rotor turbulence is dominated by rotor generated turbulence and the levels of turbulence downstream are seen to increase. In Fig. 20b where the prescribed turbulent inlet condition is at the maximum, the distribution of the kinetic energy contours is remarkably different to the low turbulence case with most of the turbulence concentrated in the left hemisphere of the rotor. This turbulence become completely dissipated with turbulent levels downstream of the rotor dropping close to zero.

These figures illustrate the effect of highly turbulent conditions on VAWT operation. In the case of the high inflow turbulence, rotor turbulence is reduced at the lee side so in that region, blade generated turbulence is dominant, whereas, in the case of low inflow turbulence, the main source of the turbulence within the rotor is blade generated turbulence regardless of the azimuthal location of the blade.

Fig. 21c shows a plot of the length scale with azimuth. Whilst not a comprehensive study on VAWT turbulence this figure shows that, for this configuration, with changes in domain inlet turbulence intensity, the average value of the length scale with azimuth tends to oscillate around a value roughly equal to the airfoil chord length $(0.183 \mathrm{~m})$. This verifies Pearson's assumption that the integral length scale of turbulence within the rotor should be set to a level somewhere in the vicinity of the airfoil's chord length when implementing

700 Amiet's inflow noise model [21. Compared to the analytical approach however, the average length scale quantity will not be sufficient for accurate calculation of this noise source.

\section{Sound Pressure Levels}

Fig. 22 shows the noise spectra for the turbine, at a standard listener location, 1.5 hub heights away from the turbine base. The results show increases in overall SPL levels for increases in turbulence intensity. As 705 turbulence intensity increases so to does noise. The location of the spectral peak for all cases is consistent. Results also lead to the conclusion that there is a saturation point that exists whereby, at a certain inlet turbulence intensity level, noise levels no longer increase.

Results of this study show that for small changes in inflow turbulence there can be a variation of up to 5 $\mathrm{dB}$ in overall sound pressure levels leading to a conclusion that for VAWT noise from inflow-turbulence noise 


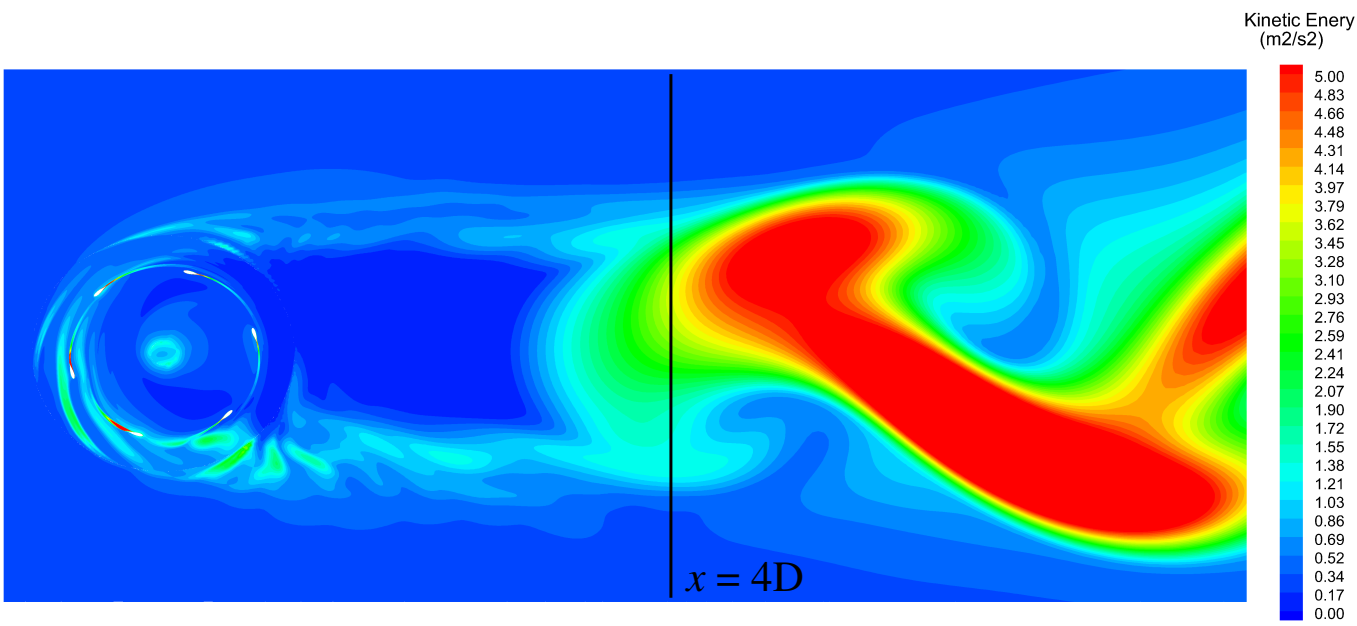

(a)

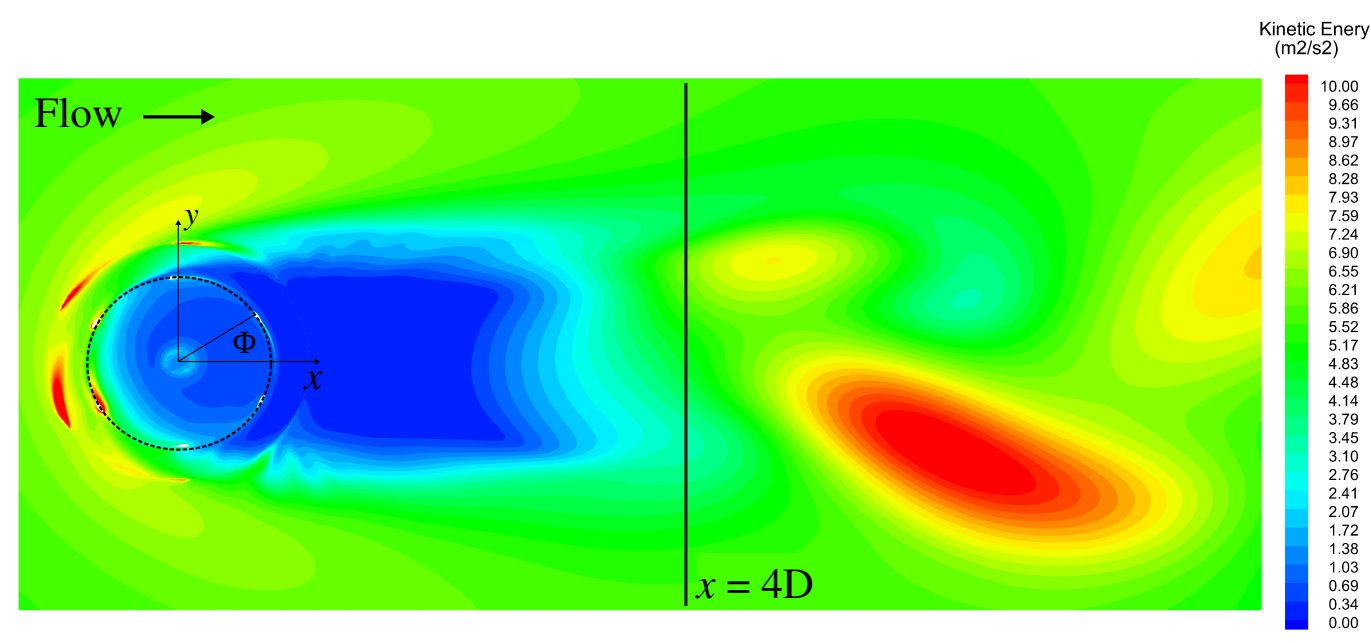

(b)

Figure 20: Turbulent kinetic energy contours with changes inflow turbulence intensity, $I_{t}$, (a) $5 \%$ (b) $20 \%$. A line is shown a position 4 diameters downstream to compare wake development at this location.

710

sources blade generated turbulence dominates the inlet turbulence. Furthermore, for accurate calculation of VAWT noise the azimuthally varying turbulence levels within the rotor need to be accurately accounted for and considered. This predicted increase in noise was made possible through the use of CFD data which showed (Fig. 21) the effect that blade rotation has in reducing the overall level of generated turbulence within the rotor compared to the inlet turbulence specification.

\section{Conclusion}

In this study a method for the prediction aerodynamically generated broadband of noise emitted by a Vertical Axis Wind Turbine was presented. Four noise prediction models were implemented to determine the levels of inflow-turbulence, and self-noise produced by the turbine. Flow parameters required by the noise models were determined either through the use of analytical models or pre-calculated CFD simulation data. By using advanced noise prediction models in conjunction with improved-accuracy flow solutions the tool provided improved results for the prediction of VAWT noise compared to existing methods. 




(a)

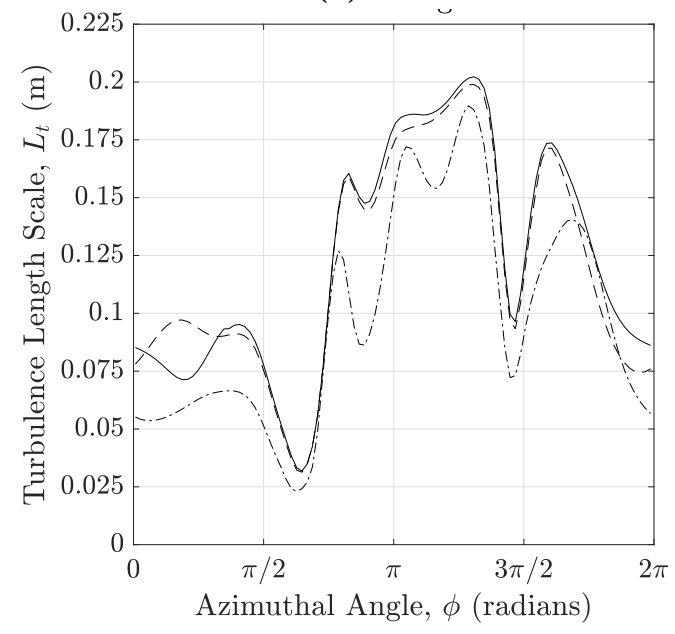

(c)

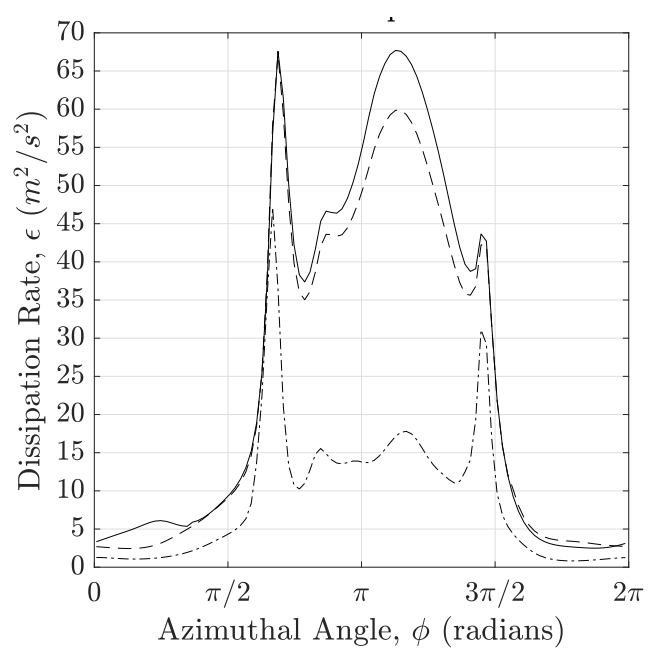

(b)



(d)

Figure 21: Turbulence parameters extracted from CFD at local blade inlet for changes in inflow turbulence intensity $\left(I_{t}\right)$ (a) turbulent velocity (b) azimuthal angle (c) turbulent length scale (d) local blade inlet velocity; $-20 \%,--10 \%,-\cdot-5 \%$

Validation of the analytical tool was performed by benchmarking prediction accuracy against measurement data for an operational VAWT. Analytical estimates for VAWT noise overpredicted a dominant inflowturbulence noise source and overprediction was attributed to poor predictions of the key input parameters - most importantly the local blade inlet velocity and secondly the angle of attack as well as less essential turbulence parameters. Airfoil self-noise models were benchmarked against individual airfoil data. The BPM model underpredicted SPL whilst the TNO model slightly overpredicted this source. Predictions for TBL noise from the QR5 matched those by Pearson [21. Both inflow-turbulence and self-noise models were also compared to calculations of Pearson where the self-noise calculations using the TNO model matched well but inflow-turbulence noise was overpredicted owing to either a commonly occurring incorrect implementation of the equations by Pearson or an overprediction of blade inlet velocity in the analytical approach.

Inflow-turbulence noise was the dominant noise source observed in the given cases. This noise source was seen to be up to $20 \mathrm{~dB}$ louder than turbulent boundary layer noise and, for VAWT's operating in urban environments, inflow-turbulence noise will continue to be the primary dominant source.

The CFD based approach was used to predict noise levels for a new six-bladed VAWT designed for the EU FP-7 SWIP project. CFD calculations provided improved blade inlet parameters compared to the 


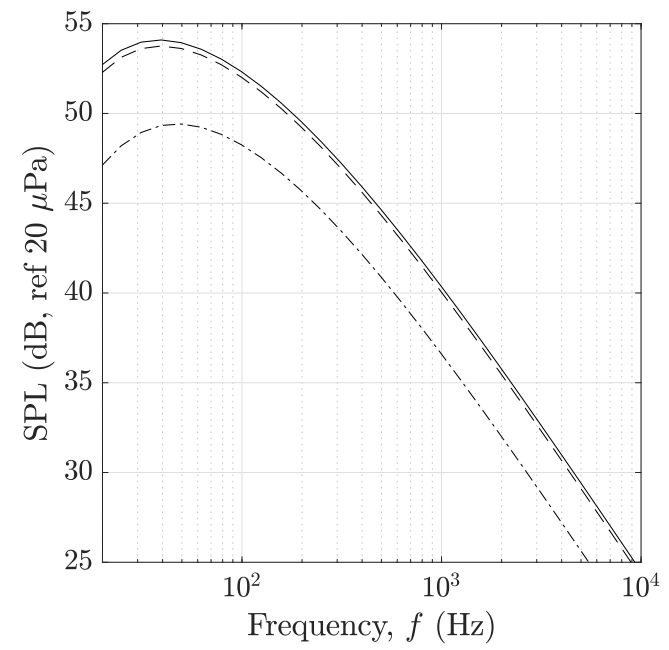

Figure 22: SPL with changes in inflow turblence intensity $\left(I_{t}\right)$ using the Paterson and Amiet model with CFD input data; - 20\%,-- $10 \%,-\cdot-$ $5 \%$

analytical baseline solution and, in particular, increased the accuracy of the important blade inlet velocity term. Ancillary information related to the turbulence within the rotor also aided in improving input data, showing that for inflow-turbulence noise sources, blade generated turbulence dominates the inflow turbulence and that for accurate calculati

This study has demonstrated a tool that can successfully predict aerodynamic noise generated by a VAWT with modest run time. This is useful for determining overall noise levels, resolving source localisation as well as running parameter studies to optimise configurations for a range of conditions particular to VAWT's operating in highly turbulent environments.

\section{Acknowledgement}

This paper and the SWIP project (New innovative solutions, components and tools for the integration of wind energy in urban and peri-urban areas) has received funding from the European Unions Seventh Programme for research, technological development and demonstration under grant agreement No. 608554 .

\section{References}

[1] J. Botha, H. Rice, A novel method of vertical axis wind turbine noise prediction, in: 10th European Congress and Exposition on Noise Control Engineering, Euronoise, Maastricht, The Netherlands, 2015.

[2] D. Marten, J. Wendler, G. Pechlivanoglou, C. Nayeri, C. Paschereit, QBLADE: an open source tool for design and simulation of horizontal and vertical axis wind turbines, International Journal of Emerging Technologies and Advanced Engineering 3 (3) (2013) 264-269.

[3] I. Paraschivoiu, Double-multiple streamtube model for studying vertical-axis wind turbines, Journal of propulsion and power 4 (4) (1988) 370-377.

[4] S. Oerlemans, J. Schepers, Prediction of wind turbine noise and validation against experiment, International journal of aeroacoustics 8 (6) (2009) 555-584.

[5] A. Rasam, J. D. Botha, K. Bolin, C. OReilly, G. Efraimsson, H. Rice, Aerodynamic noise prediction for a wind turbine using numerical flow simulations and semi-empirical modelling approaches, in: 22nd AIAA/CEAS Aeroacoustics Conference, 2846, 2016. 
[6] J. Botha, A. Rasam, D. Catháin, H. Rice, A. Shahrokhi, Some noise predictions for small wind turbines, in: Proceedings of ISMA 2016, Leuven, Belgium, 2016.

[7] J. Saab, M. de Mattos Pimenta, Displacement thickness evaluation for semi-empirical airfoil trailingedge noise prediction model, Journal of the Brazilian Society of Mechanical Sciences and Engineering 38 (2) (2015) 385-394.

[8] S. Lee, S. Lee, Numerical and experimental study of aerodynamic noise by a small wind turbine, Renewable Energy 65 (2014) 108-112.

[9] W. J. Zhu, N. Heilskov, W. Z. Shen, J. N. Sørensen, Modeling of aerodynamically generated noise from wind turbines, Journal of Solar Energy Engineering 127 (4) (2005) 517-528.

[10] T. F. Brooks, D. S. Pope, M. A. Marcolini, Airfoil self-noise and prediction, vol. 1218, National Aeronautics and Space Administration, Office of Management, Scientific and Technical Information Division, 1989 .

[11] R. W. Paterson, R. K. Amiet, Noise and surface pressure response of an airfoil to incident turbulence, Journal of Aircraft 14 (8) (1977) 729-736.

[12] G. Guidati, R. Barei, S. Wagner, An investigation of blade-tower-interaction noise (BTI) for horizontal axis wind turbines in upwind and downwind configuration. First steps towards modeling of aeroelastic effects, in: Proc. 8th IEA Symposium, 249-255, 1994.

[13] S. Buck, S. Oerlemans, S. Palo, Experimental characterization of turbulent inflow noise on a full-scale wind turbine, Journal of Sound and Vibration 385 (2016) 219-238.

[14] C. Simão Ferreira, The near wake of the VAWT: 2D and 3D views of the VAWT aerodynamics, Ph.D. thesis, TU Delft, Delft University of Technology, 2009.

[15] G. Tescione, D. Ragni, C. He, C. S. Ferreira, G. van Bussel, Near wake flow analysis of a vertical axis wind turbine by stereoscopic particle image velocimetry, Renewable Energy 70 (2014) 47-61.

[16] Q. Li, T. Maeda, Y. Kamada, J. Murata, T. Kawabata, K. Shimizu, T. Ogasawara, A. Nakai, T. Kasuya, Wind Tunnel and Numerical Study of a Straight-bladed Vertical Axis Wind Turbine in ThreeDimensional Analysis (Part I: For predicting Aerodynamic Loads and Performance, Energy 106 (2016) 443-452.

[17] R. Howell, N. Qin, J. Edwards, N. Durrani, Wind Tunnel and Numerical Study of a Small Vertical Axis Wind Turbine, Renewable Energy 35 (2010) 412-422.

[18] F. Balduzzi, A. Bianchini, R. Maleci, G. Ferrara, L. Ferrari, Critical issues in the CFD simulation of Darrieus wind turbines, Renewable Energy 85 (2016) 419-435.

[19] K. M. Almohammadi, D. Ingham, L. Ma, M. Pourkashanian, CFD Sensitivity Analysis of a StraightBlade Vertical Axis Wind Turbine, Wind Engineering 36 (2012) 571-588.

[20] SWIP Project (608554), New innovative solutions, components and tools for the integration of wind energy in urban and peri-urban areas. EU-FP7 project. Description of Work, 2014.

[21] C. Pearson, Vertical axis wind turbine acoustics, Ph.D. thesis, Cambridge University, 2014.

[22] M. Herr, R. Ewert, C. Rautmann, M. Kamruzzaman, D. Bekiropoulos, R. Arina, A. Iob, P. Batten, S. Chakravarthy, F. Bertagnolio, Broadband Trailing-Edge Noise Predictions-Overview of BANC-III Results, in: Proceedings 21st AIAA/CEAS Aeroacoustics Conference, vol. 2847, 2015.

[23] W. K. Blake, Mechanics of flow-induced sound and vibration V2: complex flow-structure interactions, vol. 2, Elsevier, 2012.

[24] R. R. Parchen, Progress report DRAW: A prediction scheme for trailing edge noise based on detailed boundary layer characteristics, TNO Institute of Applied Physics, 1998. 
[25] A. Fischer, F. Bertagnolio, H. A. Madsen, Improvement of tno type trailing edge noise models, European Journal of Mechanics-B/Fluids 61 (2017) 255-262.

[26] C. A. León, R. Merino-Martínez, D. Ragni, F. Avallone, M. Snellen, Boundary layer characterization and acoustic measurements of flow-aligned trailing edge serrations, Experiments in Fluids 57 (12) (2016) 182.

[27] O. Stalnov, C. Paruchuri, P. Joseph, Prediction of broadband trailing-edge noise based on Blake model and Amiet theory, in: 21st AIAA/CEAS Aeroacoustics Conference, 2526, 2015.

[28] S. Lee, Source Characterization of Turbulent Boundary Layer Trailing Edge Noise Using an Improved TNO Model, in: 22nd AIAA/CEAS Aeroacoustics Conference, 2812, 2016.

[29] R. K. Amiet, Acoustic Radiation from an Airfoil in a Turbulent Stream, Journal of Sound and Vibration 41 (4) (1975) $407-420$.

[30] M. V. Lowson, Assessment and prediction of wind turbine noise, Technical Report, Flow Solutions Ltd., Bristol, United Kingdom, 1993.

[31] P. Moriarty, P. G. Migliore, Semi-empirical aeroacoustic noise prediction code for wind turbines, National Renewable Energy Laboratory Golden, CO, 2003.

[32] P. A. Davidson, Turbulence: an introduction for scientists and engineers, Oxford University Press, Oxford, UK, 2015.

[33] A. P. Dowling, J. Ffowcs Williams, Sound and sources of sound, Horwood Ellis, Hemel, UK, 1983.

[34] T. F. Brooks, C. L. Burley, Rotor broadband noise prediction with comparison to model data, AIAA paper 2210.

[35] R. H. Schlinker, R. K. Amiet, Helicopter rotor trailing edge noise, in: AIAA, Astrodynamics Specialist Conference, vol. 1, 1981.

[36] S. Sinayoko, J. Hurault, On predicting wind turbine noise and amplitude modulation using Amiets theory, in: 6th International Conference on Wind Turbine Noise 2015, Glasgow, 2015.

[37] P. Fuglsang, H. Aagaard Madsen, Implementation and verification of an aeroacoustic noise prediction model for wind turbines, Technical Report, Riso DTU National Laboratory for Sustainable Energy, 1996.

[38] M. Islam, D. S.-K. Ting, A. Fartaj, Aerodynamic models for Darrieus-type straight-bladed vertical axis wind turbines, Renewable and Sustainable Energy Reviews 12 (4) (2008) 1087-1109.

[39] M. Kamruzzaman, T. Lutz, E. Kraemer, W. Wuerz, On the length scales of turbulence for aeroacoustic applications, in: 17th AIAA/CEAS Aeroacoustics Conference (32nd AIAA Aeroacoustics Conference), 2734, 2011.

[40] M. Kamruzzaman, A. Herrig, T. Lutz, W. Würz, E. Krämer, S. Wagner, Comprehensive evaluation and assessment of trailing edge noise prediction based on dedicated measurements, Noise Control Engineering Journal 59 (1) (2011) 54-67.

[41] F. Menter, Zonal two equation kw turbulence models for aerodynamic flows, in: 23rd fluid dynamics, plasmadynamics, and lasers conference, 2906, 1993.

[42] G. I. Taylor, Statistical theory of turbulence, in: Proceedings of the Royal Society of London A: Mathematical, Physical and Engineering Sciences, vol. 151, The Royal Society, 421-444, 1935.

[43] M. Drela, XFOIL: An analysis and design system for low Reynolds number airfoils, in: Low Reynolds number aerodynamics, Springer, 1-12, 1989.

[44] R. Monaghan, A Review and Assessment of Various Formulae for Turbulent Skin Friction in Compressible Flow, Aeronautical Research Council Technical Report, 1953. 
[45] P. Moriarty, G. Guidati, P. Migliore, Prediction of turbulent inflow and trailing-edge noise for wind turbines, in: Proc. of the 11th AIAA/CEAS Aeroacoustics Conf.(Monterey, CA), AIAA Paper, vol. 2881, 2005, 2005.

[46] B.-K. Dyne, S.J.C, Wind turbine noise measurements, Technical Report, ISVR Consulting, Institute of Sound and Vibration Research, University of Southampton, 2007.

[47] E. Möllerström, F. Ottermo, J. Hylander, H. Bernhoff, Noise emission of a $200 \mathrm{~kW}$ vertical axis wind turbine, Energies 9 (1) (2015) 19.

[48] A. Shahrokhi, L. Ma, D. Ingham, M. Pourkashanian, The effect of the upstream wind conditions on the performance of a vertical axis wind turbine, WindEurope (previously EWEA), 2016.

[49] P. Ma, M. Li, J. Jilesen, F. S. Lien, H. Yee, E.and Harrison, A comparison of coarse-resolution numerical simulation with experimental measurements of wind turbine aerodynamic performance, vol. 79, Procedia Engineering, 17-27, 2012.

[50] E. Sagol, M. Reggio, A. Ilinca, Assessment of two-equation turbulence models and validation of the performance characteristics of an experimental wind turbine by CFD, vol. 2012, ISRN Mechanical Engineering, 2012.

[51] F. R. Menter, S. R. Langtry, R. B.and Likki, Y. B. Suzen, P. G. Huang, S. Volker, A correlation-based transition model using local variablesPart I: model formulation, Journal of turbomachinery 128 (2006) $413-422$.

[52] E. I. Organick, A Fortran IV primer, Addison-Wesley, 1966.

[53] C. S. Ferreira, H. Bijl, G. Van Bussel, G. Van Kuik, Simulating dynamic stall in a 2D VAWT: modeling strategy, verification and validation with particle image velocimetry data, in: Journal of physics: conference series, vol. 75, IOP Publishing, 012023, 2007.

[54] A. Laratro, M. Arjomandi, B. Cazzolato, R. Kelso, Self-noise and directivity of simple airfoils during stall: An experimental comparison, Applied Acoustics 127 (2017) 133 - 146, ISSN 0003-682X.

[55] G. K. Batchelor, The theory of homogeneous turbulence, Cambridge university press, 1953.

[56] D. K. Wilson, Three-Dimensional Correlation and Spectral Functions for Turbulent Velocities in Homogeneous and Surface-Blocked Boundary Layers, Technical Report, DTIC Document, 1997.

[57] G. Tescione, D. Ragni, C. Simao Ferreira, G. van Bussel, Near wake flow analysis of a vertical axis wind turbine by stereoscopic particle image velocimetry, Renewable Energy 70 (2014) 1-15, ISSN 0960-1481.

[58] H. Lam, H. Peng, Study of wake characteristics of a vertical axis wind turbine by two- and threedimensional computational fluid dynamics simulations, Renewable Energy 90 (2016) 386 - 398, ISSN 0960-1481, URL http://www.sciencedirect.com/science/article/pii/S0960148116300118

[59] S. Oerlemans, P. Sijtsma, B. M. López, Location and quantification of noise sources on a wind turbine, Journal of sound and vibration 299 (4) (2007) 869-883. 